\title{
Winds, Waves, and Turbulence on a Shallow Continental Shelf during Passage of a Tropical Storm
}

\author{
ANN E. GARGETT \\ Institute of Ocean Sciences, Sidney, British Columbia, Canada \\ DANA K. SAVIDGE \\ Skidaway Institute of Oceanography, Savannah, Georgia
}

(Manuscript received 30 January 2020, in final form 6 March 2020)

\begin{abstract}
Measurements of collocated fields of atmospheric forcing, surface waves, and mean and turbulent velocities associated with passage of Tropical Storm (TS) Barry over the U.S. Navy Tower R2 on the Georgia continental shelf are presented. A vertical-beam ADCP enables computation of directional surface wave spectra and hence of directional Stokes functions of depth and time, as well as mean (including tidal) and turbulent velocities throughout the water column. Full-depth turbulent velocity and backscatter structures observed during TS Barry are determined to be Langmuir supercells (LS). The LS appear in the present observations and in similar observations from a shallower site only when a surface growth rate $g_{*}$ exceeds a critical value, providing a means of predicting how deep an unstratified water column must be before LS will not be expected. When observed, LS structures at Tower R2 are less organized than archetypical LS structures: we suggest that this result is due primarily to smaller near-bottom growth rate in the deeper water column. Despite $g_{*}$ values above the critical value, and appropriate values of Langmuir and Rayleigh numbers, full-depth velocity/backscatter structures disappear completely for a time between the two wind maxima associated with the TS, as wind veers rapidly clockwise with eye passage to the west of Tower R2. From the observations, the most likely explanation for this hiatus is decreased wave breaking during the period of wind veering, reducing surface supply of "effective" vertical vorticity that dominates generation of Langmuir circulation (LC). This result has significant implications for LES modeling of LC.
\end{abstract}

\section{Introduction}

Ocean modeling of turbulence and mixing associated with tropical storms or hurricanes generally uses a deepocean configuration, where it is usually concluded that mixed layer deepening is due primarily to vertical mixing occurring "as a response to the large-amplitude near-inertial currents generated within the oceanic mixed layer (OML)." (Chen et al. 2007, p. 314) although theory and large-eddy simulations (LES: Li and Garrett 1997; Sullivan et al. 2007, 2012; Kukulka et al. 2010; Liu et al. 2018; Wang et al. 2018) suggest that the presence of Langmuir circulations (LC) augments shear-generated mixing. Most in-water observations of hurricane effects on the ocean are also from the deep ocean (D'Asaro 2003; Sanford et al. 2011; Rabe et al. 2015). However many hurricanes approach land over broad shallow

Corresponding author: Ann E. Gargett, gargettann@gmail.com shelves, where storm response may differ as a result of the presence and effects of full-depth Langmuir circulations, which were first discovered in conditions of directionally constant storm winds at the Long-Term Ecosystem Observatory (LEO; a cabled observatory off New Jersey) and were named Langmuir supercells (LS) by Gargett et al. (2004). Similar conditions at Tower R2 in the South Atlantic Bight (Savidge and Gargett 2017, hereinafter SG17) produced full-depth turbulent structures that, while generally less organized than those at LEO, are nevertheless identifiable by a range of criteria as LC: here, use of the term LS will include such structures.

This paper provides the most complete description yet available of interactions among wind, wave and turbulence fields during a hurricane-like storm in shallow water, using fortuitous passage of a tropical storm (TS) over resources deployed at midshelf in the South Atlantic Bight. Pressure maps are shown in Fig. 1 at times of the 

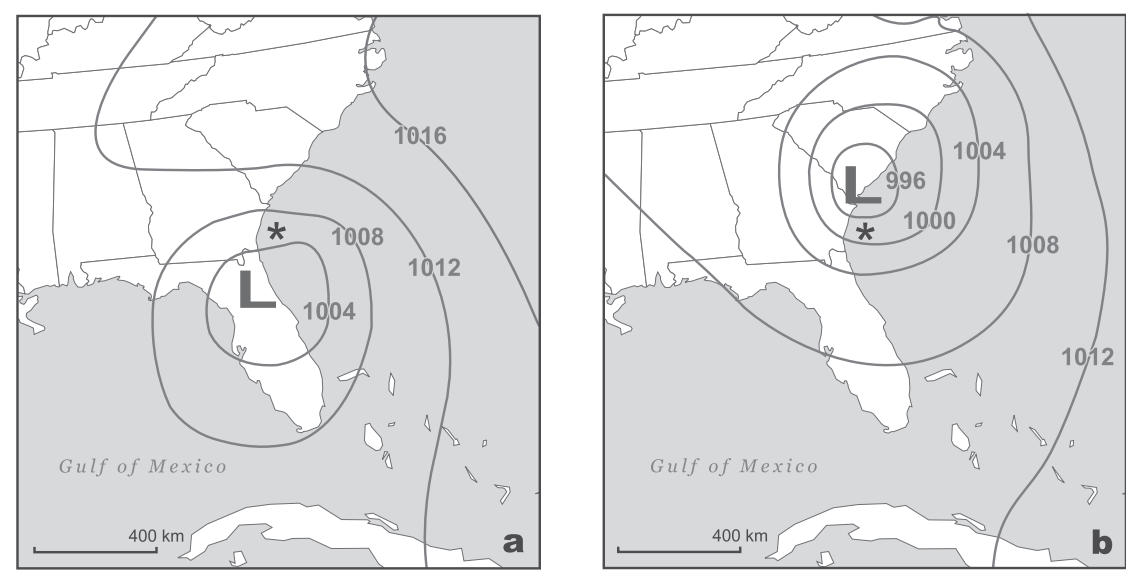

FIG. 1. Pressure fields (contours; hPa) during passage of Tropical Storm Barry over measurement site Tower R2 (star) on the Georgia continental shelf for (a) 1800 UTC 2 Jun 2007 (yearday 152) and (b) 0900 UTC 3 Jun 2007 (yearday 153), which are the times of the two wind speed maxima observed at Tower R2 (see Fig. 3, below). The fields are derived from NOAA archived maps P_10_June02_18Z.gif for (a) and P_15_June03_09Z.gif for (b).

two wind maxima observed during passage of TS Barry over the measurement location. The path of the eye is nearly south to north, so the geographic coordinate system is approximately a "hurricane coordinate system," with geographical north equal to the direction of travel. Tower R2 is situated to the east of the eye track, so winds will veer clockwise during storm passage, coming from approximately east before eye passage and rotating through north to come from approximately west afterward, as will be seen in observed vector wind time series.

\section{Data and processing}

In September 2007, the Benthic Observatory and Technology Testbed On the Mid Shelf-Understanding Processes (BOTTOMS-UP) program (Savidge et al. 2008) instrumented Navy Tower R2 $\left(31.375^{\circ} \mathrm{N}, 80.567^{\circ} \mathrm{W}\right)$, then operational in 26-m mean water depth approximately $65 \mathrm{~km}$ off the coast of Georgia. A full suite of meteorological instruments on a tower mast and a subsurface (6-m depth) pressure gauge were both part of the South Atlantic Bight Synoptic Offshore Observational Network (SABSOON) array (Seim 2000). BOTTOMS-UP added a tower-mounted temperature chain to estimate water column stratification, dominated by temperature in the South Atlantic Bight, and a five-beam verticalbeam acoustic Doppler current profiler (VADCP; slant-beam angles $30^{\circ}$ from vertical), installed $0.5 \mathrm{~km}$ northeast of the tower. Location of Tower R 2 and instruments on and near it can be found in Fig. 1 of SG17. Collocation of measured fields should be emphasized.
Data from Tower R2 are divided into sessions, each from a few to several days in length: the present work uses session 001 unless otherwise noted. Within sessions, data are divided into records of length $2 \mathrm{~h}$, and numbered sequentially: records will be referenced by number.

SG17 detail ancillary instrument data processing. In particular, surface wind stress $\tau_{*}$ is calculated as described in SG17. For the present analysis, it is also necessary to calculate bottom stress. As did Gargett and Grosch (2014, hereinafter GG14) and Gargett et al. (2014), we use the relationship $\tau_{*_{H}}=\rho_{o}\left(2.5 \times 10^{-3}\right) U_{1 \mathrm{~m}}^{2}$ (Sternberg 1968; Sherwood et al. 2006). However, while LEO provided direct measurement of $U_{1 \mathrm{~m}}$, mean velocity at $1 \mathrm{~m}$ above bottom, this must be estimated at Tower R2, where the measurement closest to the bottom is at $2.66 \mathrm{~m}$. Velocity $U_{1 \mathrm{~m}}$ was estimated by assuming a logarithmic profile, first fitting measurements of root-mean-square (over record length) total velocity from the lowest three bins (2.66-4.56 $\mathrm{m}$ above bottom) to $\ln (h)$, where $h$ is binmean height above bottom, and then extrapolating to $\ln (h=1 \mathrm{~m})$. Using medians of all records from session 001 , it was found (not shown) that $U_{1 \mathrm{~m}} \approx 0.8 U_{2.66 \mathrm{~m}}$, and this relationship is used to calculate $\tau_{* H}$.

Information on VADCP operation and beam velocity processing relevant to the Tower $\mathrm{R} 2$ deployment is also found in SG17. In brief, surface wave velocities were removed from all beam velocities by low-pass filtering, carried out over consecutive half-record lengths in order to minimize loss of near-surface vertical-beam data. Subsequent removal of linear least squares fits over halfrecord lengths results in what is here called fluctuation vertical velocity $w^{\prime}$. Since backscatter amplitude 
(a)

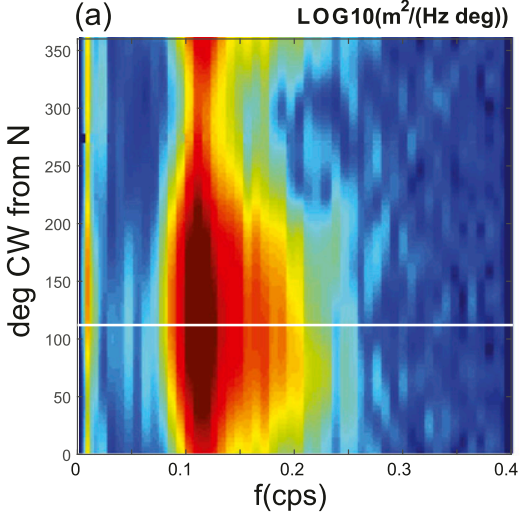

(b)

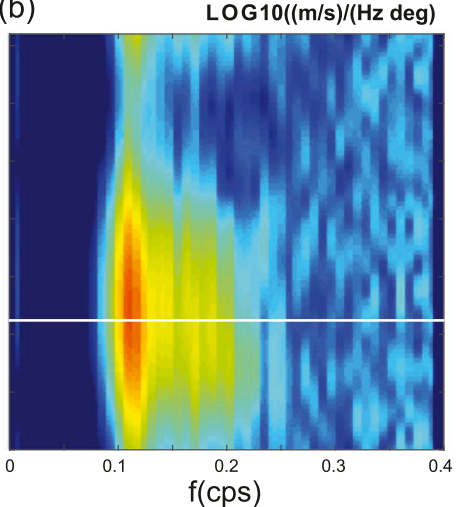

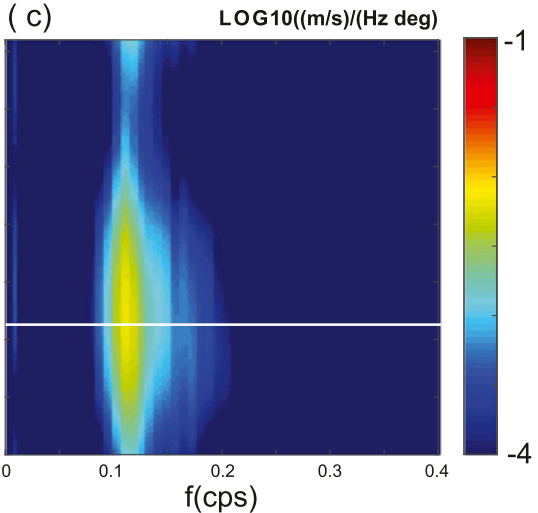

FIG. 2. (a) Logarithmic directional surface wave-height spectrum $\Phi_{\varsigma}(\omega, \theta)$ computed from VADCP beam data of record 27, at the time of the first wind speed maximum associated with TS Barry. Also shown are directional Stokes functions (b) $S\left(\omega, \theta, x_{3}=0\right)$ at the surface and (c) $S\left(\omega, \theta, x_{3}=-10 \mathrm{~m}\right)$ at $10 \mathrm{~m}$ below the surface. Direction (degrees clockwise from north) is that from which waves are propagating-over the 2 -h extent of record 27 , the average wind is from $\sim 110^{\circ}$ (white line). For computational details, see appendix A.

does not required filtering, fifth-beam backscatter A5 (corrected for beam spread and range but uncalibrated) is available right to the surface. For further details, see SG17, Gargett and Wells (2007), and Gargett et al. $(2008,2009)$.

A major addition to the data processing described by SG17 is use of VADCP data to compute directional surface wave spectra; G. Dusek and H. Seim (2011, personal communication) modified flexible open-source code that originally accepted only calculated Cartesian velocity components (Johnson 2002) to include ADCP radial beam velocities as input data. Further modification during the present analysis allows inclusion of vertical-beam data. All spectra shown here are computed over the 2-h span of data records, using 1) vertical-beam data from the bin that is both closest to the surface and available over the entire record length (i.e., below all surface wave troughs) and 2) data from all four slant beams from two bins: the shallowest bin in which sidelobe surface effects will be reliably absent, and the next deeper bin. The vertical bin is closer to the surface than the slant bins, measuring waves with smaller vertical scales: moreover addition of vertical-beam data improves the horizontal resolution associated with use of only slant beams. Appendix A provides further details of calculation of the directional surface wave-height spectrum $\Phi_{\varsigma}(\omega, \theta)$.

Because we are interested in combined wind/wave driving of turbulence as all fields evolve during storm passage, we use $\Phi_{\varsigma}(\omega, \theta)$ to derive an associated representation of Stokes drift velocity as a function of frequency, direction, and depth $\left(x_{3} \leq 0\right)$. Following Kenyon (1969), the Stokes velocity corresponding to the directional surface wave displacement spectrum is

$$
\begin{aligned}
U_{S}\left(x_{3}\right) & =\int_{0}^{2 \pi} \int_{0}^{\infty} 2 \Phi_{\varsigma}(\omega, \theta) \omega k \frac{\cos h 2 k\left(x_{3}+H\right)}{2 \sin h^{2} k H} d \omega d \theta \\
& =\int_{0}^{2 \pi} \int_{0}^{\infty} \Phi_{\varsigma}(\omega, \theta) \operatorname{St}\left(\omega, x_{3}\right) d \omega d \theta \\
& =\int_{0}^{2 \pi} \int_{0}^{\infty} S\left(\omega, \theta, x_{3}\right) d \omega d \theta,
\end{aligned}
$$

the integral over both frequency and direction of the product of $\Phi_{\varsigma}$ and a response function $\operatorname{St}\left(\omega, x_{3}\right)=$ $\omega k\left[\cosh 2 k\left(x_{3}+H\right)\right] /\left(\sinh ^{2} k H\right)$, where $k$ is calculated from wave radian frequency $\omega=2 \pi f$ using the complete dispersion relationship. In Eq. (1), the function $S\left(\omega, \theta, x_{3}\right)$ is the directional distribution of contributions to the Stokes velocity at $x_{3}$. Technically, $S\left(\omega, \theta, x_{3}\right)$ is not a Stokes spectrum because its integral is Stokes velocity, and not its square: we thus refer to $S$ as the (directional) Stokes function and will use it to identify the dominant direction of $U_{S}$ at any given depth. In place of the integral over the complete frequency range, we follow GG14 in using the integral of Eq. (1) over a standard frequency range $0.05<f<0.4$ determined by considering possible contamination of results arising through extreme response corrections at very low and high frequencies (for an extended discussion and a comparison between this and other common choices for computation of $U_{S}$, see the appendix of GG14). Figure 2 shows a sample directional surface wave-height spectrum and derived directional Stokes functions for the surface and $x_{3}=-10 \mathrm{~m}$. Record 27 is from the first velocity maximum of TS Barry, when winds from slightly south of east drove locally generated waves in the same roughly westward (shoreward) direction as that of swell initially 

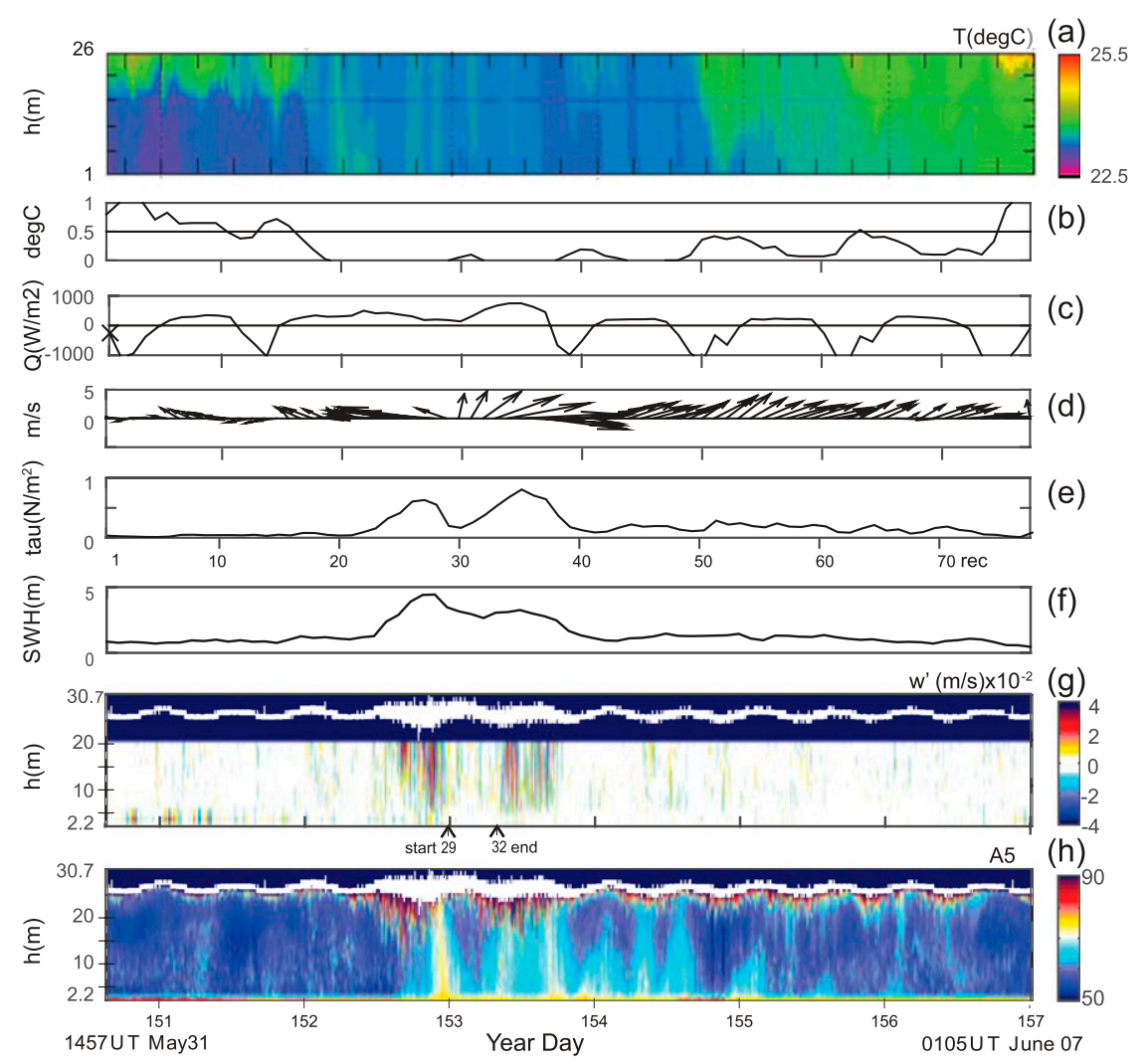

FIG. 3. (a) Water column temperature (the primary determinant of density) from the thermistor chain on Tower $\mathrm{R} 2$ as a function of height above bottom $h$, and recordaveraged values of (b) temperature difference between $\sim 1 \mathrm{~m}$ deep and $\sim 1 \mathrm{~m}$ above bottom (used as a surrogate for stratification), (c) surface heat flux (positive for ocean heat loss), (d) vector wind, (e) wind stress, and (f) SWH. Also shown are (g) fluctuation vertical velocity $w^{\prime}$ (here calculated by low-pass filtering only those bins that are available from the entire session) and (h) acoustic backscatter A5, both from the vertical beam of the VADCP. Record number (label "rec") appears at the start time of the $(2 \mathrm{~h})$ record; those annotated between $(\mathrm{g})$ and $(\mathrm{h})$ are of importance relative to wind stress (section 4).

present before storm arrival. Stokes drift functions both at the surface and $10 \mathrm{~m}$ depth have the same dominant direction (from $\sim 110^{\circ}$ ) as the surface waves and contain effectively no contribution from frequencies $<0.1$ cycles per second (cps). As expected, increasing depth decreases the magnitude of the Stokes function maximum, while higher-frequency contributions progressively disappear.

An associated Stokes shear function can be computed as the vertical derivative of Eq. (1). This function is unbounded at $x_{3}=0$ and dominated by high frequency/wavenumbers near surface: however GG14 demonstrated that it becomes bounded at high frequency/wavenumber by $x_{3}=-3 \mathrm{~m}$. This behavior was confirmed at Tower R2 (not shown): thus, as did GG14, we use shear calculated at $x_{3}=-3 \mathrm{~m}$ as a representative near-surface Stokes shear.

\section{Temporal evolution of surface forcing during TS Barry}

Figure 3 describes the temporal evolution of winds and waves throughout the TS passage. At the start of observations, the water column was stratified (mainly by temperature), winds were light and roughly from the east, and (as will be shown later) the wave field was predominantly shoreward propagating swell. There was little backscatter or fluctuation vertical velocity above noise level in the water column. Starting with approximately yearday 152.5 (record 20), as the TS moved north into the region, shoreward wind speed increased, surface wave height increased, and water column stratification disappeared (the water column is considered to be unstratified if the top-to-bottom temperature difference (Fig. $3 \mathrm{~b}$ ) is less than $0.5^{\circ} \mathrm{C}$ ). 
High backscatter appeared first near surface, then spread to the entire water column along with significant high-frequency vertical velocities. During passage of the eye of the TS to the west of Tower R2, winds veered clockwise and decreased in magnitude, while both water column backscatter and vertical velocities decreased dramatically for about $4-5 \mathrm{~h}$ (records 30-31). Significant wave height (SWH) decreased by only approximately one-third during this period, despite major change (illustrated below) in both wavenumber and direction of the surface wave field. Full-depth features in both backscatter and vertical velocity reappeared as winds subsequently increased again, now roughly from the southwest, disappearing abruptly as wind stress and SWH decreased after passage of the storm $(\sim$ record 40$)$. Wind stress and SWH remained somewhat elevated over prestorm values until the end of the period shown and high backscatter was observed in a near-surface region, with lesser values observed periodically in near-bottom regions. In this poststorm period, the upper water column becomes close to the definition of stratified during daytime periods of incoming heat flux, but reverts to unstratified at night. Fluctuation vertical velocity is near noise level $\left(\sim 0.7 \mathrm{~cm} \mathrm{~s}^{-1}\right)$ in the sampled part of the water column during this period.

Figure 4 presents time series of derived parameters used in subsequent interpretation of observations of inwater turbulence resulting from a mixture of wind, wave, and buoyancy forcings. The first, seen in Fig. 4a is a form of the growth rate characteristic of Langmuir circulations in an unstratified water column, derived by Leibovich (1977; see also Leibovich 1983) as

$$
g_{*} \equiv\left(\frac{d U_{S}}{d x_{3}} \frac{d U}{d x_{3}}\right)_{x_{3}=0}^{1 / 2},
$$

where both shears are surface values and are assumed to be directionally aligned. ${ }^{1}$ As in GG14, the vertical gradient of mean velocity is taken as $d U / d x_{3}=u_{*} / H$ and Stokes shear $d U_{S} / d x_{3}$ is evaluated at $x_{3}=-3 \mathrm{~m}$. We note that this choice differs conceptually from initial calculations of Gargett et al. (2014), which used shear calculated at $x_{3}=-0.2 H$, which is a depth of $3 \mathrm{~m}$ at LEO but $5 \mathrm{~m}$ at Tower $\mathrm{R} 2$. In retrospect, that

\footnotetext{
${ }^{1}$ This assumption fails during the second half of the storm. Since there is no applicable theory, we compute an ad hoc estimate of an "aligned" growth rate, given by $g_{*}^{a}=g_{*}(\cos \theta)^{1 / 2}$, where $\theta$ is the angle between the wind and the Stokes shear. Parameter $g_{*}^{a}$ is smaller than $g_{*}$ by $\sim 10 \%-20 \%$ for observed maximum $\theta \sim 40-50^{\circ}$. We will point out where analyses might be sensitive to this difference between $g_{*}$ and $g_{*}^{a}$.
}

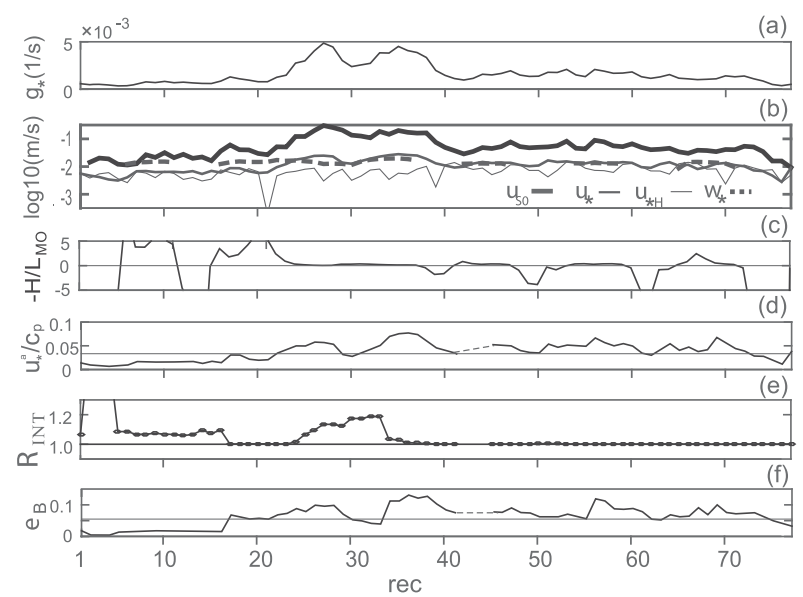

FIG. 4. Time series of derived quantities of forcing fields: (a) Langmuir circulation growth rate $g_{*}$; (b) scale velocities for Langmuir circulation $u_{S 0}$, surface stress $u_{*}$, bottom stress $u_{* H}$, and convection $w_{*}$; (c) the (negative) ratio of water depth $H$ to MoninObukhov length $L_{\mathrm{MO}}$; (d) inverse wave age, the ratio of air-side stress velocity $u_{*}^{a}$ to $c_{p}$, phase speed of the peak surface wave; (e) $R_{\mathrm{INT}}$, a single-wave indicator of wave character; and (f) $e_{B}$, the wave-breaking-threshold parameter of Banner et al. (2000). Dashed lines in wave-related parameters between records 41 and 45 remove a short period, after storm passage and before winds again increase, when a remnant wave field dominates locally windgenerated waves. Record number (rec) denotes the start of the $(2 \mathrm{~h})$ record.

choice was an unfortunate convolution of the strength of surface forcing with the effect of water column depth: calculating near-surface growth rate at the same depth, rather than the same scaled depth, provides a consistent measure of the effect of surface forcing alone. As winds and waves increase with storm arrival, $g_{*}$ rises rapidly, peaks before and after eye passage, and then decreases substantially after storm passage, although it does not return to prestorm levels until the end of the session.

Figure $4 \mathrm{~b}$ is a (logarithmic) superposition of scale velocities used for describing the strength of turbulence forced by different mechanisms. Surface Stokes velocity magnitude $u_{S 0}=U_{S}\left(x_{3}=0\right)$ characterizes wave forcing of LC. Stress velocities $u_{*}=\left(\tau_{*} / \rho_{o}\right)^{1 / 2}$ and $u_{* H}=\left(\tau_{* H} / \rho_{o}\right)^{1 / 2}$, where $\tau_{*}$ and $\tau_{* H}$ are surface and bottom stresses and $\rho_{o}$ is a characteristic mean water density, characterize turbulence driven respectively by surface and bottom stresses. The convective scale velocity $w_{*}=\left(B_{o} H\right)^{1 / 3}$ is nonzero only when surface buoyancy flux $B_{o}$ is destabilizing, that is, when $B_{o}>0$ can drive unstable oceanic convection. Values of $u_{S 0}$ climb steeply at the beginning of the storm and remain elevated relative to the other three until the end of the record. Although there is heat loss, hence destabilizing buoyancy flux, throughout the storm (Fig. 3c), Fig. 4c shows that the ratio of depth $H$ to Monin-Obukhov 
length scale $L_{\mathrm{MO}} \sim 0$ at this time, so convection does not dominate stress in forcing the boundary layer (values of $-H / L_{\mathrm{MO}}>5$ are required for convective dominance in the atmospheric boundary layer; Holtslag and Nieuwstadt 1986). Moreover in section 4 we show that stress forcing of turbulence is much weaker than Langmuir forcing during the storm period. Thus, although there is no reason why these simple scale velocities should reveal forcing dominance, they appear to be an effective predictor, at least when differences approach an order of magnitude.

Three additional fields describe various features of the surface wave fields that drive LC. In Fig. 4d, inverse wave age $u_{*}^{a} / c_{p}$, the ratio of air-side stress velocity $u_{*}^{a}$ to the phase speed $c_{p}$ of the peak wave exceeds $1 / 30$ (horizontal line), the traditional criterion for developing seas, as the storm arrives and remains elevated until near the end of the session. Figure $4 \mathrm{e}$ is $R_{\mathrm{INT}} \equiv \cosh 2 k_{p} H /\left(2 \sinh ^{2} k_{p} H\right)$, the ratio of surface Stokes velocity of a single (peak) wave in water depth $H$ to that of the same wave in deep water, used by Gargett et al. (2014) as an indicator of wave type: $R_{\mathrm{INT}}=1.0$ (horizontal line) is characteristic of deep-water waves and as $R_{\mathrm{INT}}$ increases, wave character changes first to intermediate, then to shallow-water type. Original waves were of shallow-water/intermediate type, but in record 17 change abruptly to deep-water type as storm winds generated local seas. These seas evolve to intermediate type in the first wind maximum, but by this metric transition rapidly back to deep-water type as the changing wind direction beat down existing wind seas before beginning to build up waves in the new wind direction following eye passage. Figure $4 \mathrm{f}$ shows a wavebreaking criterion proposed by Banner et al. (2000), $e_{B}=H_{p} k_{p} / 2$, where $k_{p}$ is the wavenumber of the displacement spectral peak and $H_{p}$ is the SWH of "dominant" waves, defined as those within $30 \%$ of the peak. Banner et al. (2000) define a critical value of 0.05 (horizontal line) above which breaking occurs: this value is first exceeded around record 16, as nearsurface backscatter appears in the vertical beam (Fig. $3 \mathrm{~h}$ ). The $e_{B}$ peaks during both wind speed maxima; during eye passage, it falls to critical in record 29 and remains below critical during records 30,31 , and 32 . It hovers just above critical during the poststorm period, when near-surface bubble clouds persist.

On the basis of the observed and derived fields of Figs. 3 and 4, the extent of tropical storm Barry is taken as records 20-40. Figure 5 shows frequency spectra of the wave field for a 48 - $\mathrm{h}$ period encompassing the storm. As winds increase starting in record 20, locally forced waves increase in magnitude and decrease in frequency, reaching a first wave-height peak (Fig. 3f) in records

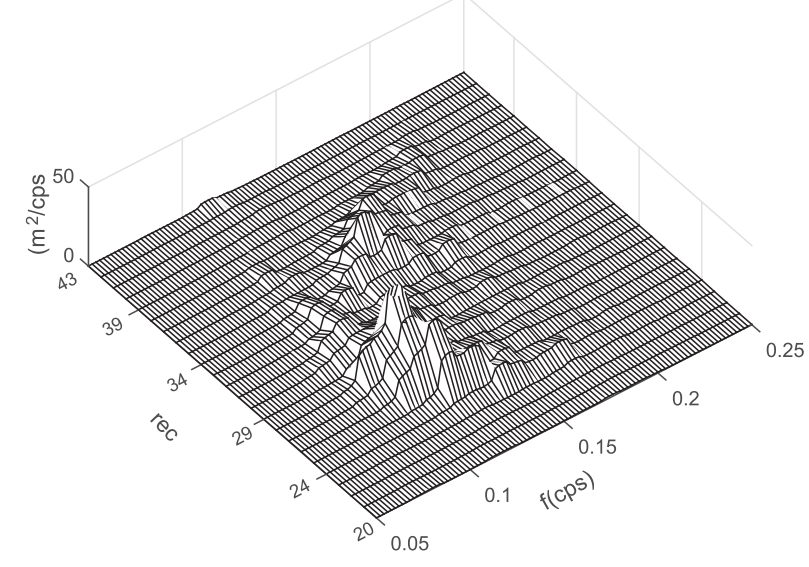

FIG. 5. Surface wave-height frequency spectra as a function of record number for a 48-h time period during passage of TS Barry. The frequency spectrum is the directional integral of the directional wave spectrum computed from VADCP beam velocities.

27 and 28: at this point, waves are of intermediate character by the metric of Fig. 4e. As winds subsequently decrease in magnitude with eye passage, then rapidly switch direction while rising to a second maximum associated with the trailing half of the TS, the original wave peak fades and a secondary peak grows in at higher frequencies, before itself fading away as winds drop after the storm. The "new" waves, those generated in the trailing part of the TS after eye passage, remain deep-water waves by the simple metric of Fig. 4e and never reach the height of the "old" waves generated in the first wind maximum (Fig. 3f), despite somewhat higher maximum wind stress (Fig. 3e) in this second period.

In Fig. 6, thumbnail plots of (base-10 logarithms of) full directional wave spectra reveal the complex behavior of the surface wave field during passage of the TS in more detail. (To avoid discontinuity in direction over $0^{\circ} / 360^{\circ}$ as winds (and waves) change direction during storm passage, the directions shown are those the waves are coming from: the direction from which the recordaveraged wind is blowing is the horizontal white line.) During storm approach, wave-height growth in the direction of the wind (roughly aligned with preexisting shoreward-propagating swell) is seen clearly through record 28. Wind direction begins to change in record 29, reaching a second stable direction (from $\sim 230^{\circ}$ ) by $\sim$ record 36 . The wave field follows the wind, but with a substantial time lag: at the time of the second peak in wind stress (records 35 and 36), peak waves remain significantly to the left of the wind. As the storm departs and wind speed falls, decrease in magnitude of the new wave field eventually reveals the continued presence of the old lower-frequency wave field. After record 41, the 


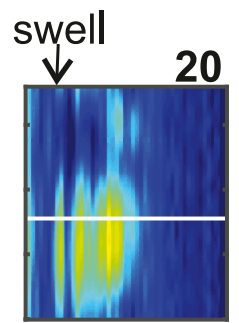

26

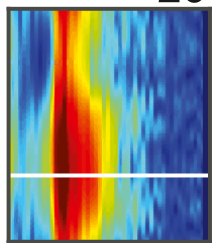

32

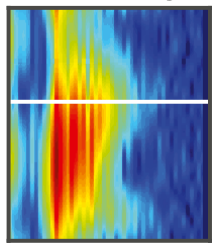

38

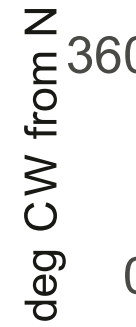

0

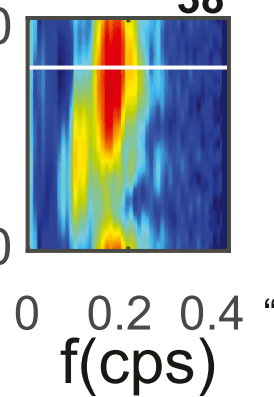

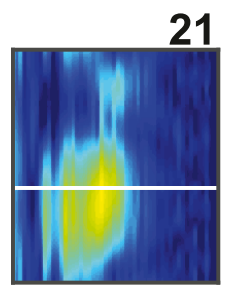

27

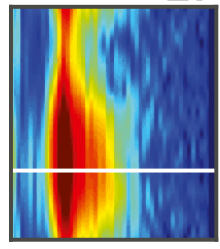

33

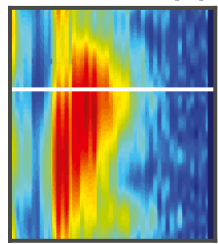

39

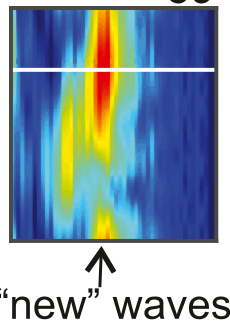

"new" waves

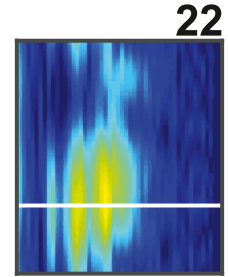

28

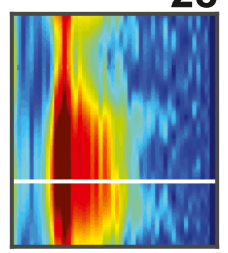

34

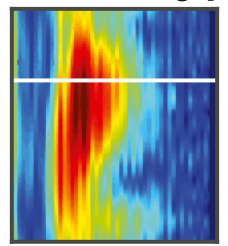

40

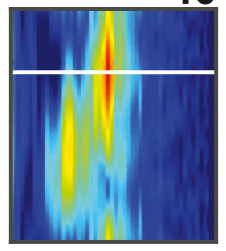

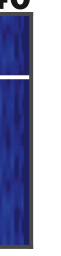

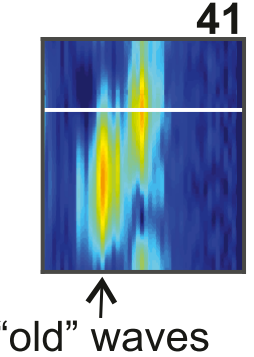

23

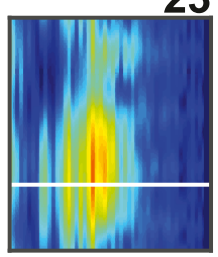

29

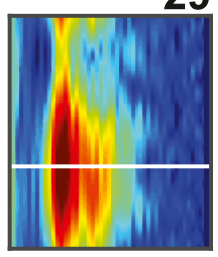

35

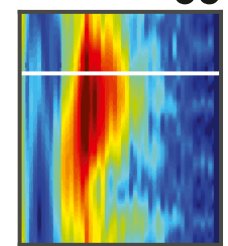

41

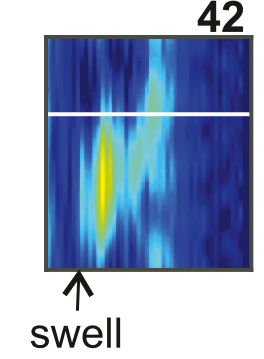

24

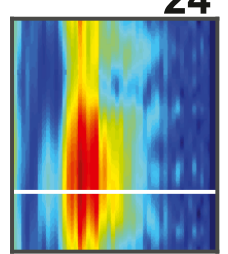

30

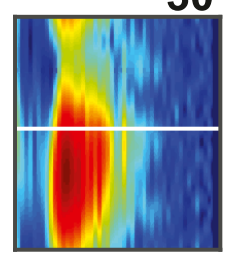

36

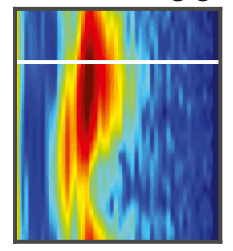

42

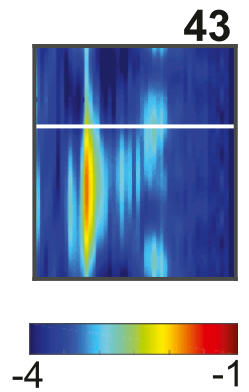

FIG. 6. Base-10 logarithms of directional surface wave-height spectra, computed from VADCP beam data as described in appendix A. The full color bar denotes $\log \left[\mathrm{m}^{2}\left(\mathrm{cps}^{\circ}\right)^{-1}\right]=[-4,-1]$. Directions are those from which waves are propagating. A horizontal white line marks the direction from which record-average wind is blowing. The frequency of swell present before the storm arrived is marked on record 20: the swell peak reemerges as the storm departs (see record 42). The peak frequency of new waves-those formed in the direction of the second wind maximum-is marked on record 39: as these fade with storm departure, old lower-frequency waves formed in the direction of the original wind maximum emerge, becoming dominant after record 41 .

magnitude of the old field can exceed that of the new. This pattern of a single wave system in the leading half of a tropical low, with multiple wave systems in the trailing half, resembles that described for hurricanes by Hwang and Walsh (2018).

The directional surface wave spectra of Fig. 6 can be used to calculate directional Stokes functions at any depth: Figs. $7 \mathrm{a}$ and $7 \mathrm{~b}$ show the surface Stokes function $S_{0}=S\left(f, \theta, x_{3}=0\right)$ and a "deep" Stokes function $S_{H}=$ $S\left(f, \theta, x_{3}=-20 \mathrm{~m}\right)$, calculated near the bottom of the water column. The maximum of $S_{0}$ starts slightly off the direction of the wind, but quickly becomes centered on the direction of the wind as this increases in magnitude. During the rapid change in wind direction, the direction of the peak of the surface Stokes function lags the wind, becoming closely aligned again only in record 38. By this time, wind stress magnitude has decreased to near prestorm levels and the surface Stokes function associated with the new wave field subsequently decays in magnitude, eventually revealing a low-frequency peak associated with the old wave field.

The behavior of the deep Stokes function $S_{H}$ differs from $S_{0}$ in some expected ways, being smaller in magnitude (note order of magnitude decrease in scale of Fig. 7b), with diminished high-frequency content. Major differences in time development of $S_{H}$ relative to $S_{0}$ are (a) delayed increase in magnitude of $S_{H}$ at the start of the event and (b) earlier decrease in magnitude at event end. 
(a)

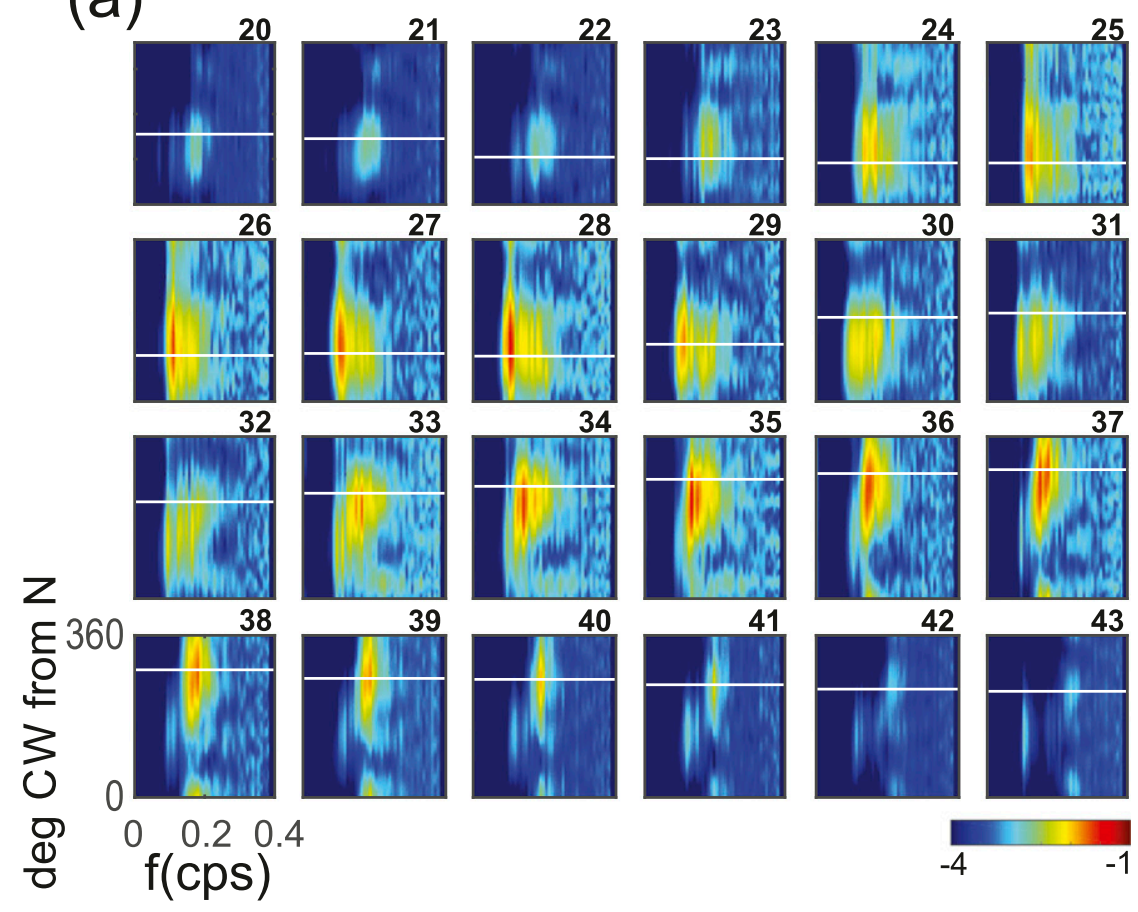

(b)
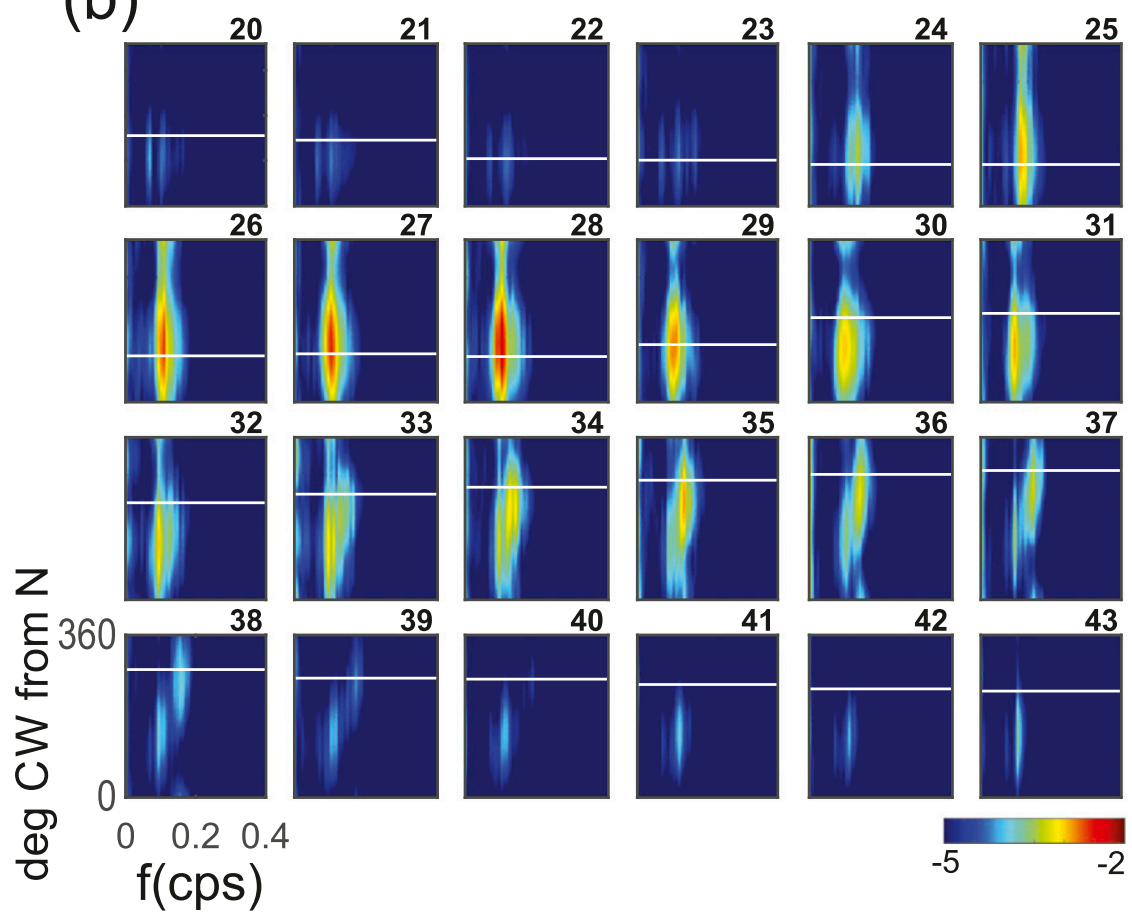

FIG. 7. Base-10 logarithmic plots of Stokes functions evaluated from the directional wave spectra of Fig. 6 (a) at the surface $S_{0}=S\left(f, \theta, x_{3}=0\right)$ and (b) near the bottom $S_{H}=S\left(f, \theta, x_{3}=-20 \mathrm{~m}\right)$. For the direction convention, see the caption to Fig. 6. 
These features are consistent with (a) finite time required after wind onset for waves to develop from initially short wavelengths (high frequencies) to the longer wavelengths (frequencies) that have significant Stokes drift velocity at depth and (b) faster decay of short wavelength waves as local wind forcing decreases. More subtle differences, both temporal and with depth, are better appreciated in Fig. 8, which shows the time evolution of magnitude (Fig. 8a) and dominant direction (Fig. 8b) of Stokes velocities at depths of $x_{3}=0,-5,-10,-15$, and $-20 \mathrm{~m}$. [Dominant direction for the Stokes velocity at any depth can be determined from the two-dimensional Stokes function $S(f, \theta)$ in one of two ways, either 1) by identifying the peak of $S_{\theta}(\theta)$, the integral of $S$ over $f$, or 2) by forming $S_{f}(f)$, the integral of $S$ over $\theta$, identifying its peak frequency $f_{p}$, and then finding $\theta$ corresponding to the maximum of $S\left(f_{p}, \theta\right)$. We chose the former method, because the latter proved noisier in cases with weak waves and/or more than one wave train.] Fig. 8c displays directional differences, plotted so that the directional difference between surface Stokes vector velocity $\mathbf{U}_{S 0}$ and wind $\mathbf{U}_{w}$ is negative when $\mathbf{U}_{S 0}$ lies to the left of the wind. Differences between $\mathbf{U}_{S 0}$ and $\mathbf{U}_{S 0}\left(x_{3}\right)$ are positive for deep values to the left of the surface value. Because it is unlikely that differences less than $\sim 20^{\circ}$ are significant (directional resolution of the Stokes function is $2^{\circ}$ but the function is smoothed over 3 points in both frequency and direction), this region is shaded.

During the first half of the storm, Stokes velocities are in the same direction as the wind from top to bottom of the water column (marked wind-aligned in Fig. 8c). In record 29 , the wind begins a rapid clockwise rotation (Figs. 3d and 8b), "leaving behind" the Stokes velocities. Through records 30 through $34, \mathbf{U}_{S 0}$ lies significantly $\left(40^{\circ}-50^{\circ}\right)$ to the left of the new wind direction, while deeper in the water column, Stokes velocities lie progressively to the left of $\mathbf{U}_{S 0}$ by (marginally significant) amounts that increase with depth. By the second wind maximum in record 35, Stokes velocities at all depths have realigned with $\mathbf{U}_{S 0}$, which however remains $\sim 40^{\circ}-50^{\circ}$ to the left of the wind. Although the near-bottom Stokes drift associated with these new waves is smaller than that of the old waves in the first wind maximum (Fig. 8a), it is significantly different from zero.

As the TS departs, wind stress falls (Fig. 3e) and the new seas decay (Fig. 5). The algorithm for dominant direction begins to identify the old wave field as dominant, first at 20-m depth in record 39 and then at progressively shallower depths to the surface in records $40-43$.

\section{Time evolution of turbulence during TS Barry}

Features of the fluctuation vertical velocity field seen in Fig. $3 g$ during passage of TS Barry, that is, full water
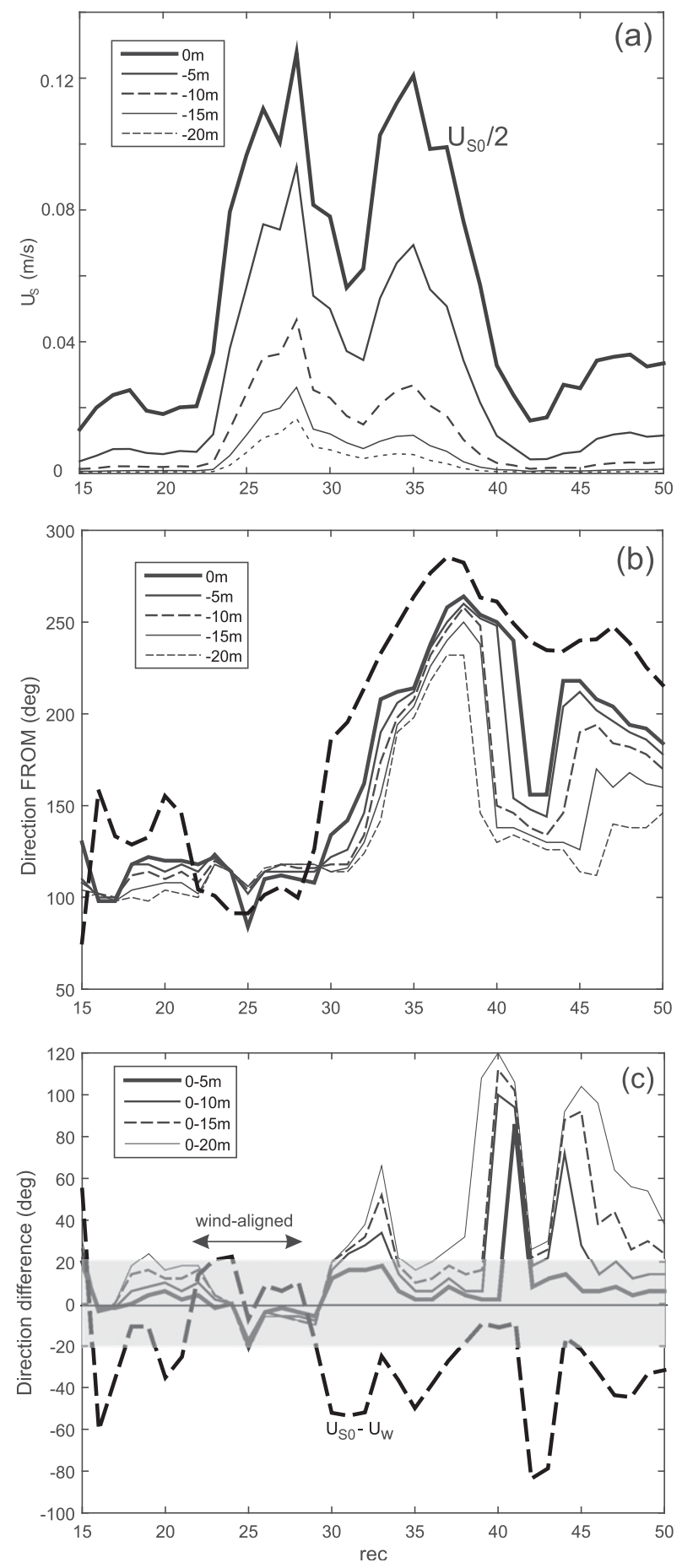

FIG. 8. (a) Magnitude of the Stokes velocity $U_{S}$ at $x_{3}=0,-5,-10$, -15 , and $-20 \mathrm{~m}$ for records including TS records 20-40 (for range compression, the surface Stokes velocity magnitude is divided by 2 ). (b) Direction of the wind velocity $\mathbf{U}_{w}$ (heavy dash) and dominant directions of the Stokes velocities at $x_{3}=0,-5,-10,-15$, and $-20 \mathrm{~m}$. Directions indicate from. (c) Differences in directions between the surface Stokes velocity $\mathbf{U}_{S 0}=\mathbf{U}_{S}\left(x_{3}=0\right)$ and the wind velocity $\mathbf{U}_{w}$ (heavy dash: negative difference implies that the vector $\mathbf{U}_{S 0}$ is to the left of the wind), and between $\mathbf{U}_{S 0}$ and deeper Stokes velocities (positive values imply deep values to the left of $\mathbf{U}_{S 0}$ ). Values outside the shaded area are considered to be significantly misaligned. 
column structures present during both wind stress maxima but absent for a period of some hours after the rapid wind direction change that begins in records 28 and 29, raise two important questions. First, are the turbulent structures observed during TS Barry waveforced LS? Second, what causes the disappearance of these structures during eye passage?

To examine the first question, consider two scaling parameters defined by GG14. Their Langmuir number $\mathrm{La}=u_{*} / u_{S 0}$ is the ratio of the surface stress velocity to the surface Stokes velocity and scales the magnitude of inertial to Langmuir vortex forces in the turbulent momentum equation. Assuming that density is a linear function of temperature $T$ only, their Rayleigh number $\mathrm{Ra} \equiv\left(\alpha_{T} g k_{T}^{-1}\right)\left(Q t_{*}^{2}\right)\left[\alpha_{T}\right.$ is the coefficient of thermal expansion, $g$ is the acceleration of gravity, $k_{T}$ is the thermal conductivity, $Q$ is the surface heat flux defined as positive when destabilizing to the ocean, and $t_{*}=g_{*}^{-1}$ is the time scale associated with the LC growth rate of Eq. (2)], scales the magnitude of buoyancy (heat) to inertial forcing. In the LEO dataset, LS were associated with $\log (\mathrm{La})<0.5$ and $\log (|\mathrm{Ra}|)<\sim 5.5$, that is, when both Langmuir forcing is sufficiently larger than and buoyancy forcing is sufficiently smaller than inertial (stress) forcing. In Fig. 9b, La is seen to fulfill the first requirement throughout hurricane passage: values remain near the threshold both before and after. For clarity, Fig. 9a displays only those records with $\mathrm{Ra}>0$, that is, where heat flux was destabilizing. Rayleigh number is above its threshold when storm winds commence in record 20 but drops substantially below the threshold in record 24, coincident (Fig. 9c) with increase in $\overline{\left\langle w^{\prime 2}\right\rangle}$, which is the vertical velocity variance formed as an average over time (angle brackets) and depth (overbar). This transition is also seen in the regime diagram of Fig. 10, where values of $\overline{\left\langle w^{\prime 2}\right\rangle}$ are generally enhanced for records within the LS region of the regime diagram. Note, however, that records during eye passage ( 30 and 31 ) show no signs of LS and have $\overline{\left\langle w^{\prime 2}\right\rangle}$ near noise level, indicating that position within this region is a necessary but not always sufficient condition for the existence of LS. ${ }^{2}$

Based on relative surface forcing magnitudes as described by the $\{\mathrm{La}, \mathrm{Ra}\}$ parameter space, the answer to the first question posed above is that when full-depth velocity structures are seen during TS Barry, they are indeed LS. However, as was the case under a storm at R2 with steady wind direction, detailed by SG17, the present LS structures are significantly less organized than archetypical

\footnotetext{
${ }^{2}$ Use of $g_{*}^{a}$ in place of $g_{*}$ increases $\log (\mathrm{Ra})$ by $\sim 0.1-0.2$. This could move record 31 just above the regime boundary in Fig. 10, but record 30 would remain within the LS region.
}
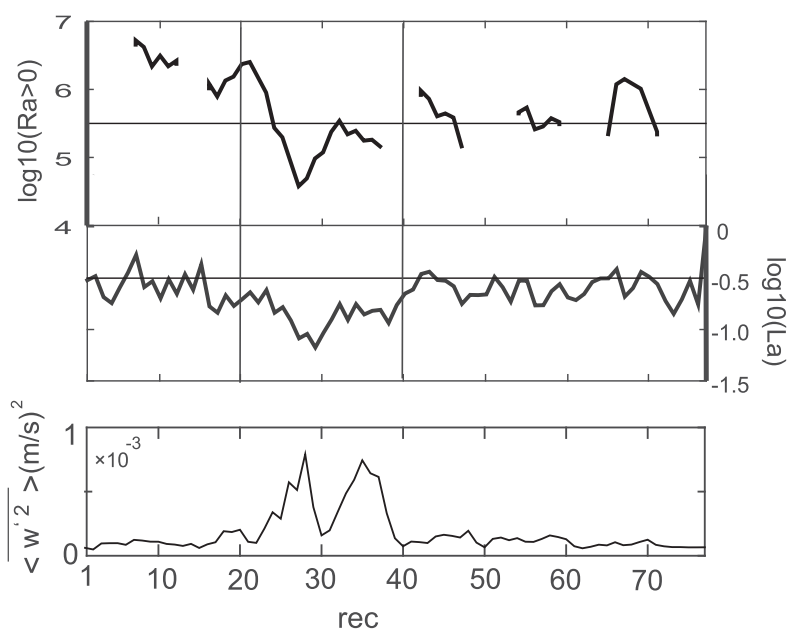

FIG. 9. Time series of (logarithms of) scaling parameters defined by GG14 and used to identify surface forcing that produces LS. (top) Rayleigh number Ra at times when surface heat flux is destabilizing $(Q>0)$. (middle) Langmuir number La. Vertical lines denote the approximate extent of TS Barry. Horizontal lines in each panel denote thresholds below which GG14 reported LS; (bottom) $\overline{\left\langle w^{\prime 2}\right\rangle}$, the record (time) and vertically averaged vertical velocity variance, is diagnostic of turbulence in the water column. Record number (rec) appears at the start of the $(2 \mathrm{~h})$ record.

LS structures observed at LEO (Gargett et al. 2004), which characteristically have downwelling/upwelling associated with bottom-intensified maximum/minimum in downwind velocity and divergent/convergent crosswind flow near bottom. Figure 11 shows highly smoothed fluctuation velocity fields in wind coordinates for three records during TS Barry. Record 28 is at peak onshore wind stress during the approach of the storm, 31 during the lull in wind speed magnitude and change in wind direction during eye passage to the west, and 35 at the second wind stress peak. While there are individual examples of the defining structure expected of organized LS in the records taken at maximum wind stress (marked in Figs. 11a and 11c), many structures lack either one or both of the features associated with LS down/upwellings at LEO. Scores have been developed to quantify degree of "organization" based on these two characteristics (appendix B) and, with the exception of records 28 and 35, structures observed during the TS (Fig. B2b) have both scores distinctly lower than those for the archetypical LS structures (Fig. B2a), that is, downwellings are less likely to have associated downwind velocity and less likely to have a change of sign of crosswind velocity near bottom. Since this is also true during directionally steady wind events at Tower R2 (Fig. B2c; see also SG17), less organized structure is not primarily due to the nonsteady nature of TS winds but characteristic of all LS at Tower R2. Thus a general question becomes why full-depth LS are less organized at Tower R2 than 


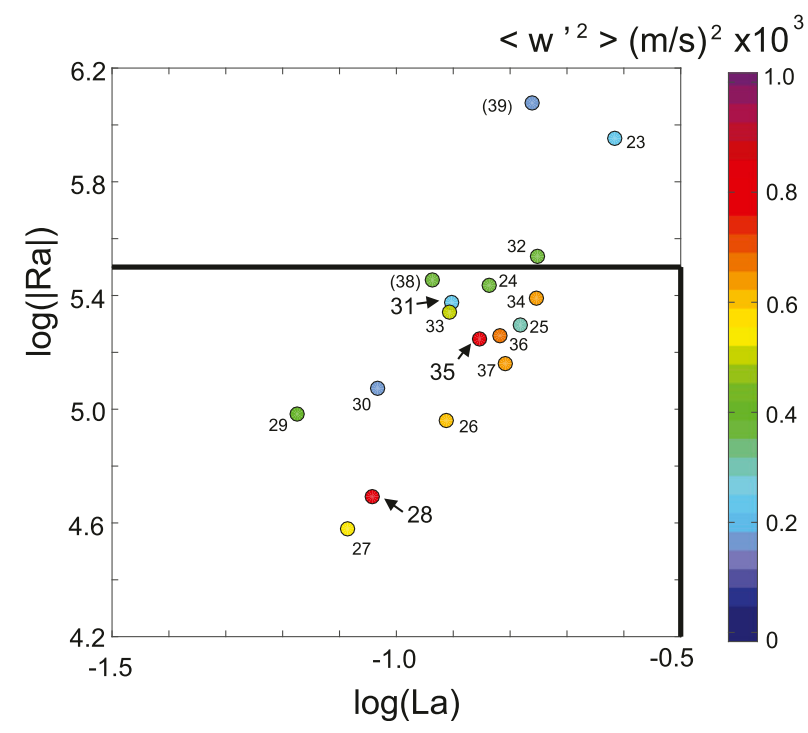

FIG. 10. Regime diagram for TS records near or within the region $\log (\mathrm{La})<0.5$ and $\log (|\mathrm{Ra}|)<5.5$ (delimited by thick lines) that was found to contain LS at LEO (GG14). Records before 23 and after 39 have $\log (|\mathrm{Ra}|)>6.2$ and/or $\log (\mathrm{La})>-0.5$. Records 38 and 39 (in parentheses) are the only ones with stabilizing surface heat flux. The colors code the value of $\overline{\left\langle w^{\prime 2}\right\rangle}$, vertical velocity variance averaged over record length in time (angle brackets) and available water column depth (overbar). Larger numbers highlight those records with velocity fields shown in Fig. 11, below.

LEO, despite falling within the "LS range" of surface forcing parameters $\mathrm{La}$ and $\mathrm{Ra}$.

A tentative answer to this question may lie in differences in LC growth rates at LEO and Tower R2. The near-surface growth rate $g_{*}$ of Eq. (2) is calculated using "surface" shears. Based on the idea that Langmuir forcing can occur not just at the surface but wherever there is both a nonzero, vertically sheared Stokes drift velocity and a source of vertical vorticity (such as the turbulent bottom boundary layer), a near-bottom growth rate $G_{*}$ can be defined in a similar way:

$$
G_{*}=\left(\frac{d U_{S}}{d x_{3}} \frac{d U}{d x_{3}}\right)_{x_{3} \simeq-H}^{1 / 2} .
$$

Since Stokes shear goes to zero at $x_{3} \equiv-H$ (see the analytical form in the appendix of GG14), it must be evaluated close to but not at the bottom; hence $x_{3} \simeq-H$ in Eq. (3). For the same reason discussed with respect to the surface calculation, we calculate Stokes shear at fixed height above bottom $(2 \mathrm{~m})$ rather than a scaled height. Near-bottom shear is here scaled as $\left(d U / d x_{3}\right)_{x_{3} \simeq-H}=u_{*_{H}} / H$, which differs from Gargett et al. (2014), who assumed that bottom stress was equal in magnitude to surface stress, that is, that $\left(d U / d x_{3}\right)_{x_{3} \simeq-H}=u_{*} / H$. While this is a good approximation at LEO (Fig. 12a), observed bottom stress at Tower R2 is substantially smaller than surface stress during LS events, as seen for both the TS and an event with quasi-constant wind stress in Figs. 12b and $12 \mathrm{c}$, respectively. (Note that for reasons of dynamic range, Fig. 12 presents stress velocities: since stress is proportional to stress velocity squared, the difference in stresses is substantially larger.) The fact that $u_{* H}<u_{*}$ at Tower R2 combines with the increase in depth by a factor of roughly 2 over that at LEO to make the mean near-bottom shear scale much smaller. In addition, provided surface wave fields do not differ enormously, a deeper water column has substantially less Stokes shear near the bottom than a shallow one. Both effects combine to make $G_{*}$ at Tower R2 much smaller than that at LEO, as seen in Fig. 13. [For clarity, Fig. 13 labels individual records only from the archetype LEO event (squares) and from the TS event that is the focus of this paper (circles). The ellipse shown contains LS records from the LS event at Tower R2 that is described in detail by SG17. Similar results are found, although they are not shown, for the two other LEO LS events described in Gargett et al. (2014) and for other LS events at Tower R2.] It was initially expected that there would be some threshold in $G_{*}$ below which full-depth fluctuations would not appear, as near-bottom Stokes shear became too small to efficiently rotate small-scale vertical vorticity, generated in the bottom boundary layer, to horizontal. Instead, using data from both relatively steady wind-wave forcing as well as the unsteady case of TS Barry, Fig. 13 illustrates a clear threshold in $g_{*}$ : if $g_{*}>g_{*}^{\text {crit }} \cong 2 \times 10^{-3}\left(\mathrm{~s}^{-1}\right)$, full depth structures in turbulent velocities and backscatter amplitude exist in unstratified water columns.

The existence of this threshold provides a method for calculating how deep a water column must be before surface-wave-forced structures do not penetrate the full depth of an unstratified water column. Knowing only $u_{*}$, from measurement or estimate of wind speed at $10-\mathrm{m}$ height, and enough information about the surface wave field to calculate Stokes shear at 3-m depth, fulldepth structures will not be expected in water of depth greater than

$$
H^{\text {crit }} \simeq\left(g_{*}^{\text {crit }}\right)^{-2}\left(\left.u_{*} \frac{d U_{S}}{d x_{3}}\right|_{x_{3}=-3 \mathrm{~m}}\right) .
$$

Existence of full-depth structures does not, however, ensure that such structures are organized. We postulate that once the surface growth rate threshold is exceeded, hence full-depth structures exist, the degree of organization of these structures depends on $G_{*}$ through the time scale $G_{*}^{-1}$ associated with near-bottom growth rate relative to the time scale(s) of mean flow variation. Different 


\section{(a)}

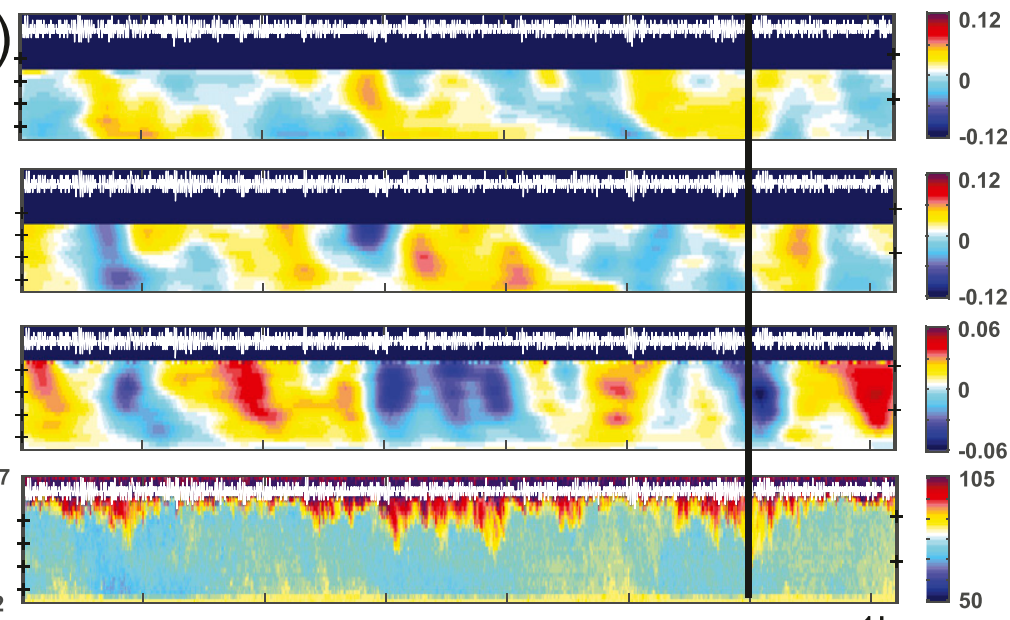

$1 \mathrm{~h}$

(b)
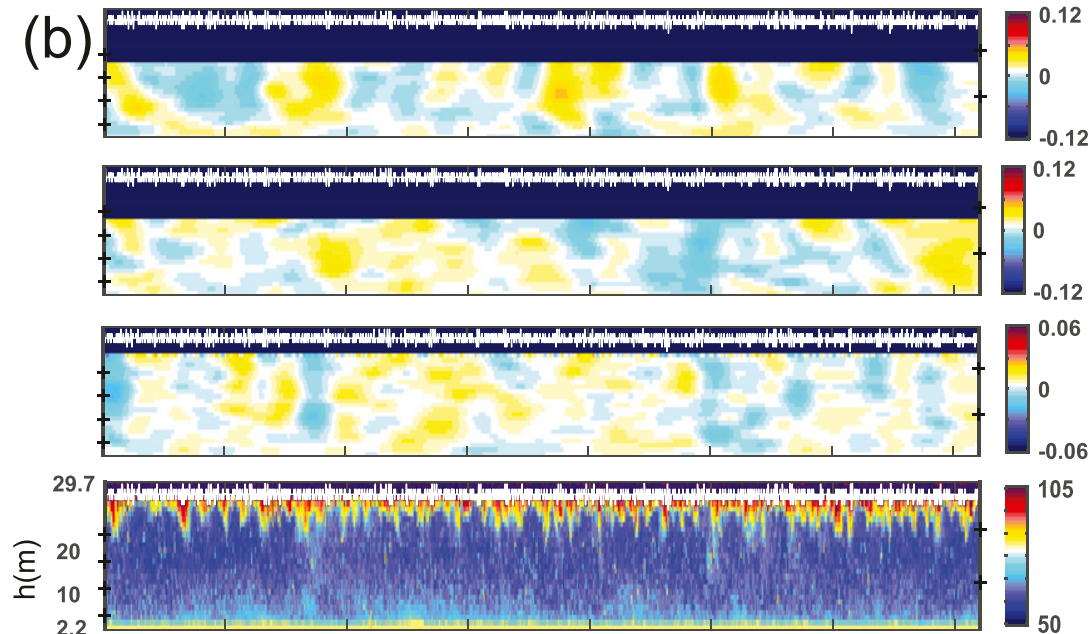

$1 \mathrm{~h}$

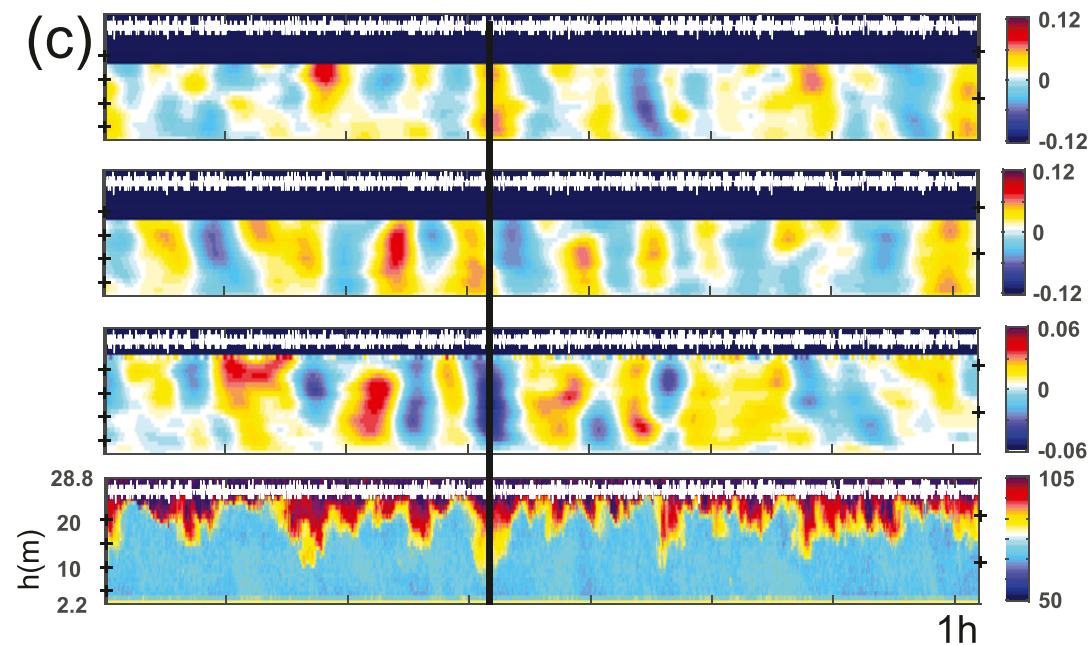

FIG. 11. Fields of fluctuation velocities in wind coordinates for three records during TS Barry. In each labeled section, the top plot is $u$ (positive in the wind direction), the topmiddle plot is crosswind velocity $v$, and the bottom-middle plot is vertical velocity $w$ (positive upward): 1-s velocities have here been heavily smoothed (by Hanning filters over 
near-bottom growth rates (typically $\sim 5 \times 10^{-4} \mathrm{~s}^{-1}$ for LEO and $\sim 1 \times 10^{-4} \mathrm{~s}^{-1}$ for Tower R2) yield growth time scales of only $\sim 30 \mathrm{~min}$ for LEO, but $\sim 3 \mathrm{~h}$ for Tower R2. Taking these as characteristic of times over which ordered structure can emerge, LS are clearly more likely at a location with an LC growth period short compared to the dominant time scale(s) over which mean flow changes, which is the semidiurnal tidal period at both sites. We thus hypothesize that ordered structures spanning the entire water column emerge frequently at LEO because the near-bottom growth time there is sufficiently shorter than semidiurnal. More tentatively, we speculate that structures are less ordered at Tower $\mathrm{R} 2$ predominantly because near-bottom growth time there is comparable to that of the semidiurnal tide. This may be the limiting factor on near-bottom organization, rather than greater disruption due to larger magnitude tidal currents at Tower R2, or any of the other possibilities discussed in SG17.

The remaining question, specific to the TS data, is why full-depth circulations effectively disappear during records 30-31 (Fig. 3), despite the facts that both records fall within the LS range of surface forcing parameters (Fig. 10), have LC scale velocities far in excess of other turbulent scale velocities (Fig. 4b), and both surface and near-bottom growth rates comparable to records with LS (Fig. 13).

Given the complex nature of changes in wind speed and direction, surface wave field, and associated Stokes velocity profiles, various reasons for the disappearance of LS can be suggested, though as wave-driven features, any explanation must in some way involve changes in the surface wave field (or the wind, in so much as it drives the waves). Comparisons between wind/wave forcing characteristics of records which have full-depth circulations (LS records) can be used to eliminate many potential "reasons" for the observed lack of such circulations in the quiescent records (30 and 31): we consider the following.

1) Change in $S W H$. Figure $3 f$ documents a relative minimum in SWH during the quiescent records; however the magnitude of SWH in the minimum is similar to that at times with LS, for example, records 25 and 38, suggesting that change in SWH alone is not a dominant factor.
2) Change in surface wind stress. Wind stress $\tau_{*}$ has a substantial minimum in records 29 and 30 (Fig. 3e), that is, approximately $2 \mathrm{~h}$ in advance of the records with minimum $\overline{\left\langle w^{\prime 2}\right\rangle}$. While full depth structures disappear during the decrease in wind stress in record 29, they do not reappear until record 33, well after the subsequent increase in wind stress in the second half of the storm, suggesting that change in wind stress magnitude alone is not of dominant importance.

3) Excessive difference in direction between wind stress and surface Stokes drift. Figure 14b provides an enlarged view of the directional differences during storm records $20-40$ (seen for the entire session in Fig. 8c), along with the time behavior of $\overline{\left\langle w^{2}\right\rangle}$ superimposed on backscatter hung from the instantaneous surface. Neither the maximum magnitude of $\overline{\left\langle w^{2}\right\rangle}$ (Fig. 14a) nor the degree of organization of structures (Fig. B2b) are noticeably different when wind and $\mathbf{U}_{S 0}$ are aligned in the first wind/wave maximum of the storm or unaligned by $40^{\circ}-50^{\circ}$ in the second maximum. Thus directional difference between the wind and $\mathbf{U}_{S 0}$ appears to be by itself irrelevant to the presence/absence of full-depth LC in cases with comparable magnitudes of wind/wave forcing. However when combined with marginal forcing magnitudes during the quiescent records, directional difference may prove important (see point 5 below).

4) Large directional shear in the Stokes drift profile. As seen in Fig. 14b, Stokes velocity in records $30-31$ is aligned within $\sim 20^{\circ}$ over depths from the surface to nearly $20 \mathrm{~m}$, comparable to the alignment in LS record 35. Moreover record 33, which has the largest directional shear during the TS, nevertheless has LS. It thus seems that a Stokes drift profile that is sheared to the extent observed here also does not inhibit full-depth LC.

5) Change in LC growth rates. Although records 30 and 31 have the minimum values of $g_{*}$ seen during TS passage (Fig. 3), as seen in Fig. 13 those values remain close to those for LS records during the TS, in particular record 24, and comparable to those from LS records under quasi-steady wind/wave forcing (ellipse). However if these values of $g_{*}$ are modified to allow for an angle of $40^{\circ}-50^{\circ}$ between the wind and

\footnotetext{
$\longleftarrow$

4 min and three vertical bins) to show larger structures more clearly. The bottom plot shows unsmoothed backscatter amplitude, revealing the range of scales present. In each plot, a white line indicates instantaneous surface as determined from vertical-beam backscatter. Shown are records (a) 28, at peak onshore wind stress during the approach of the storm, (b) 31 , during the lull in wind speed magnitude and changing wind direction during eye passage to the west of Tower R2, and (c) 35, at the second (roughly offshore) wind stress maximum. The vertical lines in (a) and (c) highlight archetypical LS structures.
} 

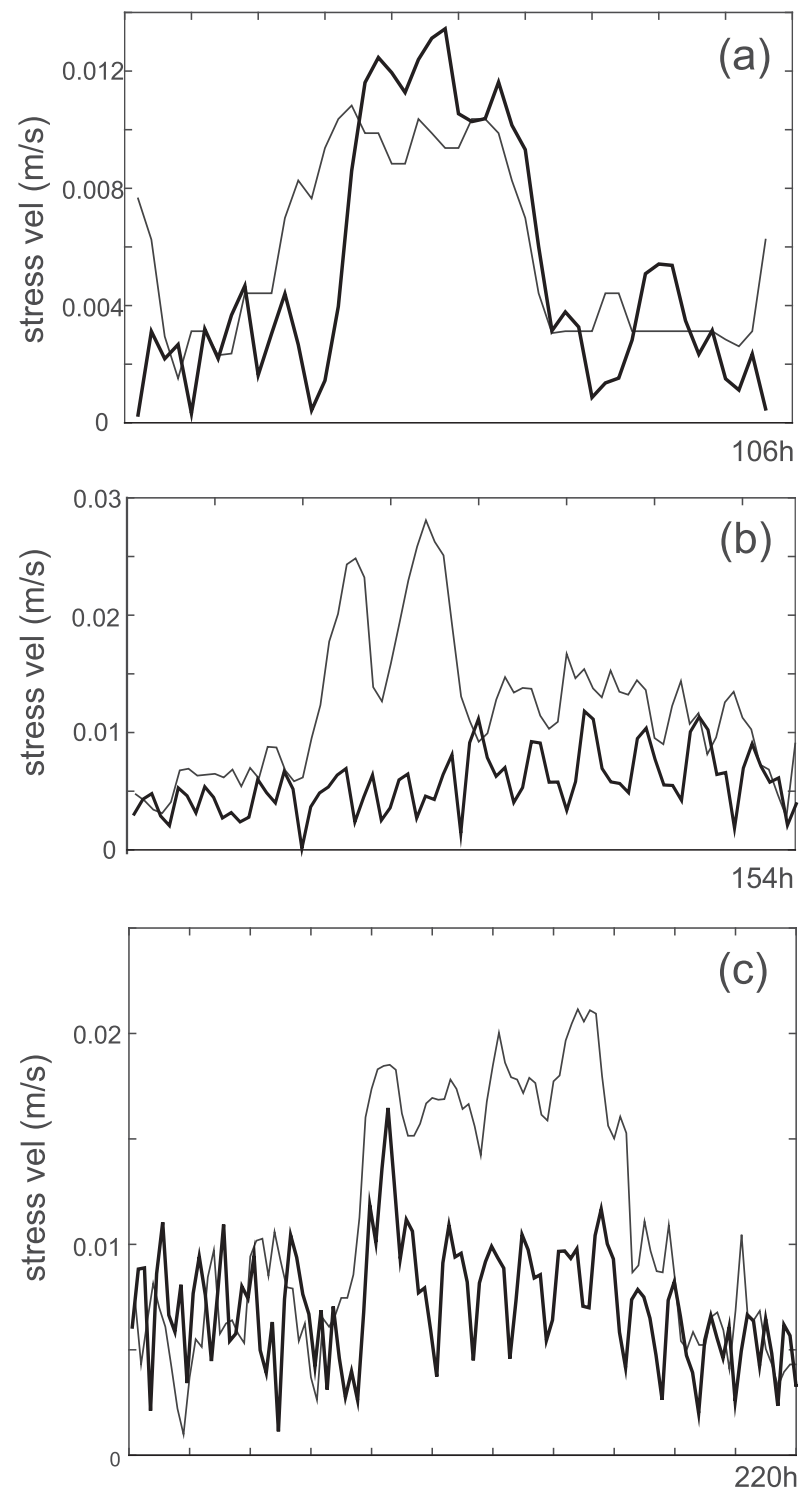

FIG. 12. Stress velocities at the surface ( $u_{*} ;$ thin line) and $2 \mathrm{~m}$ above bottom $\left(u_{*_{H}}\right.$ thick line) for (a) the archetypical LS event at LEO (session 043) and two Tower R2 periods: (b) TS Barry (session 001) and (c) an LS event with relatively constant wind/wave fields (portions of sessions 017 and 018; see SG17). In storm conditions, bottom stress is approximately equal to surface stress at LEO but is typically significantly smaller than surface stress at Tower R2.

Stokes shear at $x_{3}=-3 \mathrm{~m}$ during records 30 and 31 (Fig. 14b shows that Stokes velocities are aligned over $0-5-\mathrm{m}$ depths and hence Stokes shear at $x_{3}=-3 \mathrm{~m}$ is aligned with $\mathbf{U}_{S 0}$ : we thus use the angular differences between $\mathbf{U}_{S 0}$ and $\mathbf{U}_{w}$ shown in Fig. 14b), the magnitudes decrease to $0.8-0.9$ of the values shown, thus falling nearer the critical threshold of $g_{*}^{\text {crit }} \cong 2 \times 10^{-3}\left(\mathrm{~s}^{-1}\right)$. It is thus possible that decrease in growth rate due to directional differences between wind shear and

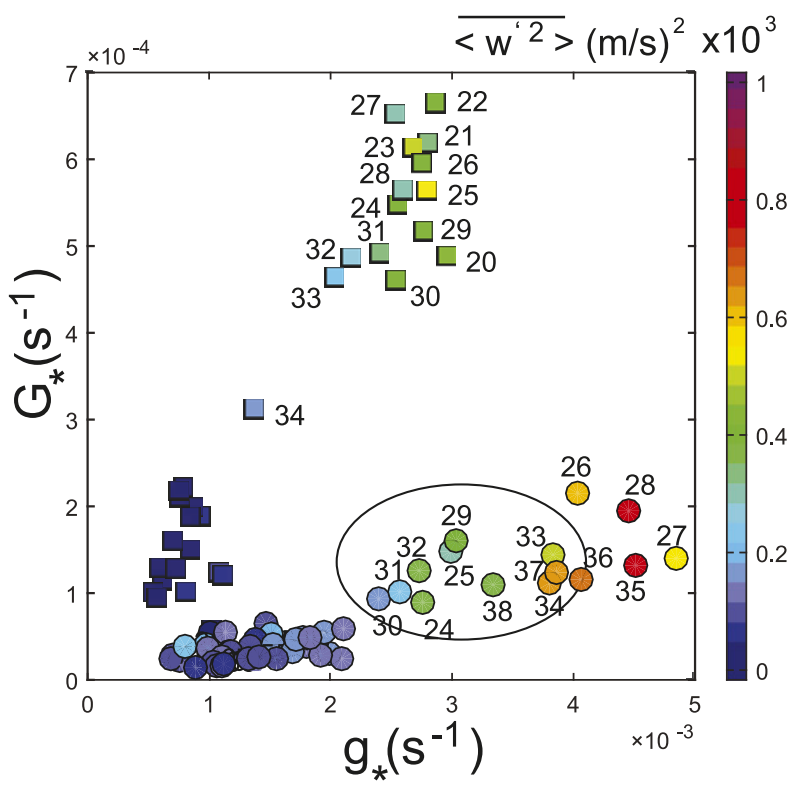

FIG. 13. Near-bottom LC growth rate $G_{*}$ vs near-surface LC growth rate $g_{*}$ for unstratified records. Squares are from a supercell event at LEO (session 043), and circles are from the TS event at Tower R2 (session 001). In both cases, records with full-depth vertical velocity and backscatter signatures are labeled by number. For a supercell event at Tower R2 (session 017-018; see SG17), all such records lie within the ellipse shown: for clarity, individual points for records in this event are not plotted. Records are color coded by value of $\overline{\left\langle w^{\prime 2}\right\rangle}$.

Stokes shear, when coupled with decreased magnitude of both surface wind stress and Stokes shear, could explain absence of LS during records 30 and 31. This explanation would imply a very sharp criticality at $g_{*}^{\text {crit }}$ and moreover one separating a state with LS from a state with no LC, rather than from a state with LC over a depth range less than full depth. Although such a sharp criticality cannot be dismissed (and indeed if true would be a very significant result), we think it unlikely.

6) Decrease/cessation of surface wave breaking. Absence of LS in the quiescent records could be associated with decrease in surface wave-breaking activity during this time period, as evidenced by the relative absence of surface-origin backscatter in records 30 and 31 (Figs. 3, 11b, and 14a), and by the fact that $e_{B}$ drops below the critical value of 0.05 during records 30-32, while exceeding it substantially in both wind maxima (Fig. 4f). It must be admitted that Gargett et al. (2014) provide at least one example when this metric does not satisfactorily predict onset of breaking as evidenced by surface-origin backscatter. However, in the present case, the general behavior of $e_{B}$ is at least consistent with an intuitive picture in which, allowing for reasonable time lags, the veering of the wind that 
(a)
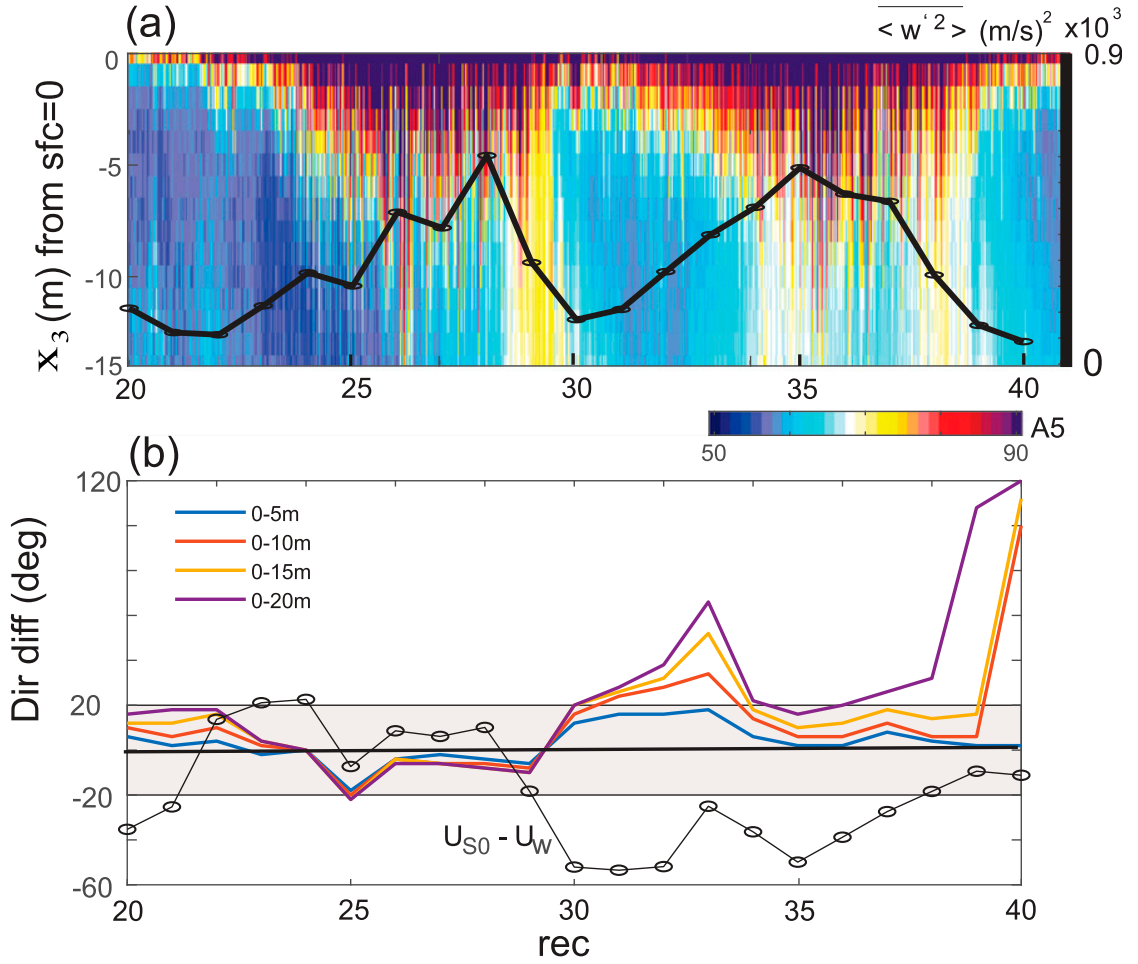

FIG. 14. (a) Color shades showing vertical-beam backscatter A5, hung from the instantaneous surface. Superimposed are record-averaged values of $\overline{\left\langle w^{12}\right\rangle}$. (b) An enlargement over storm records $20-40$ of directional differences seen in Fig. $8 \mathrm{c}$ for the entire session; $U_{S 0}-U_{w}$ $<0$ implies that $\mathbf{U}_{S 0}$ is to the left of the vector wind). Positive values of difference between $\mathbf{U}_{S 0}$ and deeper Stokes velocities imply deep values to the left of $\mathbf{U}_{S 0}$. Values outside the shaded area are considered to be significantly misaligned. In (a) and (b), symbols mark the start of each record.

starts in records 28 and 29 first acts to decrease the slope of old waves, inhibiting their breaking by record 30 , and then eventually builds up new waves to breaking levels. A reason why changes in wave breaking could have a profound effect on the formation of LC may be found in a figure that Leibovich (1977, his Fig. 4) first presented to illustrate torques involved in LC generation by the Craik-Leibovich (Craik and Leibovich 1976) CL2 mechanism. Assuming unidirectional Stokes vertical shear, he depicts a small-scale spanwise (direction normal to the Stokes shear) perturbation of the surface velocity parallel to the Stokes shear. Although Leibovich does not identify it as such, the generic form he used is that expected from a spilling breaker (Sullivan et al. 2004, 2007). Any such quasi-symmetric perturbation is associated with paired vertical vorticity that, rotated to horizontal by the Stokes shear, would efficiently generate the paired vorticity associated with LC. The small-scale surface velocity/vorticity perturbations required for the CL2 mechanism are generally assumed to be those associated with the wind stress-driven turbulent boundary layer. However in classical boundary layer turbulence, vorticity structures tend to be random in orientation, so only a fraction of the available vertical vorticity contributes to LC generation. In contrast, velocity perturbations associated with spilling breakers (of whatever scale) are roughly symmetric and aligned in the direction of the waves, hence of the Stokes drift, ideal for effective generation of LC.

\section{Inertial response}

Limited observations available under hurricanes in deep water (D'Asaro 2003; Sanford et al. 2011) find strong inertial oscillation generated on the right side of a cyclone as a partially resonant response to clockwise veering of the wind during hurricane passage. These inertial oscillations cause the shear instability at the mixed layer base that is believed responsible for a major part of the mixed layer deepening and surface layer cooling associated with hurricanes in deep water (Chen et al. 2007). What evidence is there for the generation of inertial response in the shallow ocean? 

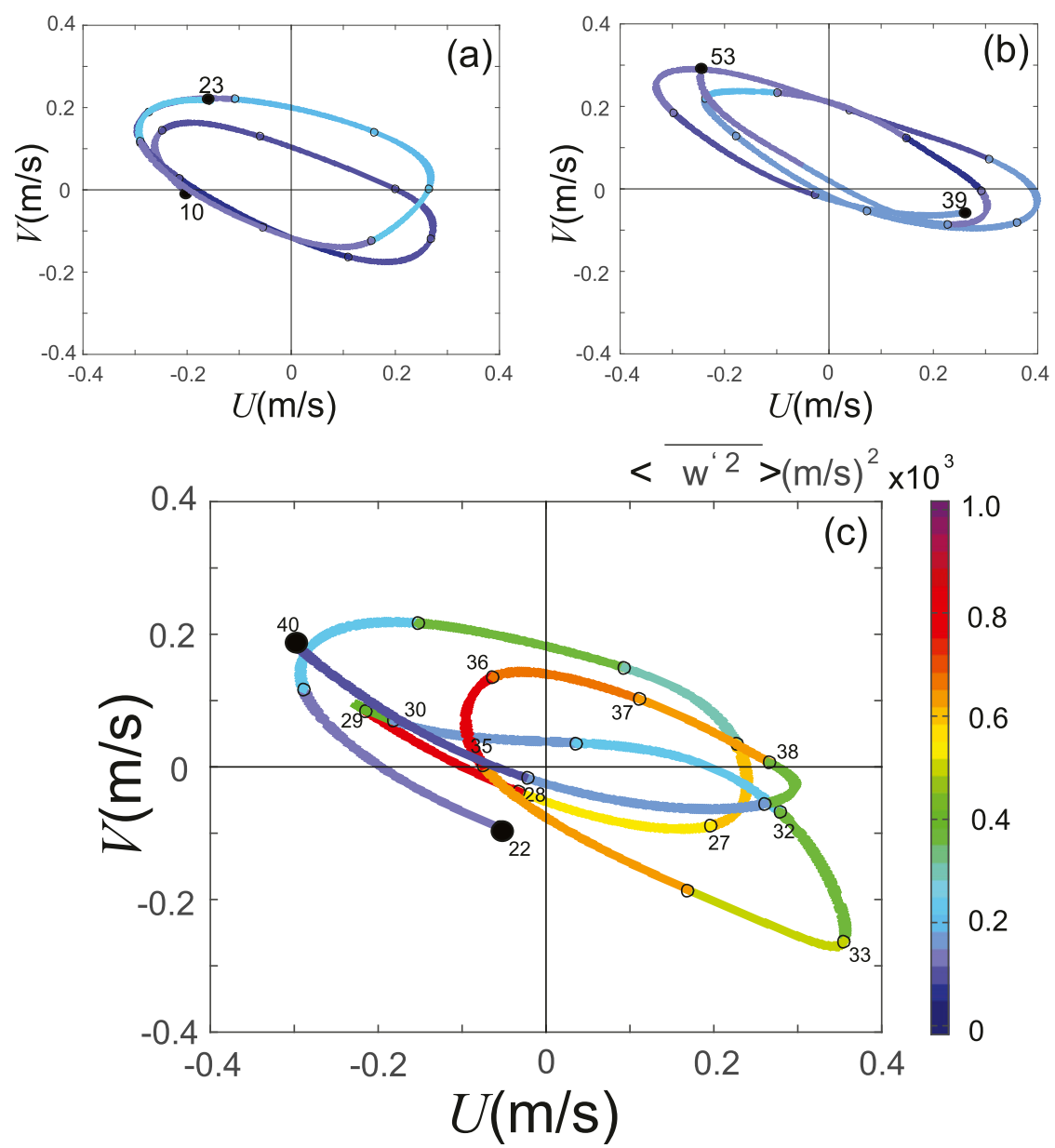

FIG. 15. Hodographs of VADCP-derived mean plus tidal velocities in geographic coordinates for periods (a) before arrival of TS Barry (records 10-23), (b) after departure of the storm (records 39-53), and (c) records during the storm (22-40). Open circles denote the start of a 2-h record; filled circles denote the start of the first record and the end of the last record. Line segments are color coded by value of $\overline{\left\langle w^{12}\right\rangle}$ for the record.

The inertial period of $\sim 13 \mathrm{~h}$ at Tower $\mathrm{R} 2$ is sufficiently close to the semidiurnal tidal period that separation in frequency space is not feasible: moreover separation by rotational spectra is not possible since both waves rotate clockwise. Instead, in Fig. 15 we examine hodographs of mean plus tidal velocities from a middepth $(14.1 \mathrm{~m}$ above bottom) bin for periods before, during, and after the storm. Figure 15a shows two circuits of a (neap) tidal ellipse centered on zero: mean currents were small before storm arrival. Afterward (Fig. 15b), the tidal ellipse has become larger (in progression toward spring tides) and is now offset roughly to the NE by a poststorm mean current, seen clearly in progressive vector diagrams (PVDs) of Fig. 16. During the storm, careful examination of Fig. 15c shows a tidal ellipse similar to that of Fig. 15a that is abruptly deformed as the wind veers rapidly clockwise in records 28 and 29 . This deformation of the tidal ellipse seems likely due to inertial motion at approximately the semidiurnal period of the tide, with magnitude $U_{i}$ of order $\sim 0.1-0.2 \mathrm{~m} \mathrm{~s}^{-1}$ (needed to "stall" the tide in record 29). Note that deformation lasts clearly for only one, at most two, inertial periods, vanishing by record 40 . This observation is consistent with a rough upper bound estimate of damping time scale $T_{i} \sim U_{i} H / u_{* H}^{2}$, made assuming linear variation of bottom stress from $\tau_{* H}=\rho_{o} u_{* H}^{2}$ at $x_{3}=-H$ to zero at the surface and neglecting internal stresses due to turbulence. With the observed value of $u_{* H} \sim 0.005 \mathrm{~m} \mathrm{~s}^{-1}$ during the TS (Fig. 12b), $T_{i} \sim 1.2-2.4$ days for $U_{i} \sim 0.1-$ $0.2 \mathrm{~m} \mathrm{~s}^{-1}$. Thus, as observed on a deeper shelf by MacKinnon and Gregg (2005), while the physics that acts to generate inertial motion in the deep ocean also does so in the coastal ocean, in the latter environment inertial motion is quickly damped out. Not surprisingly, loss of 

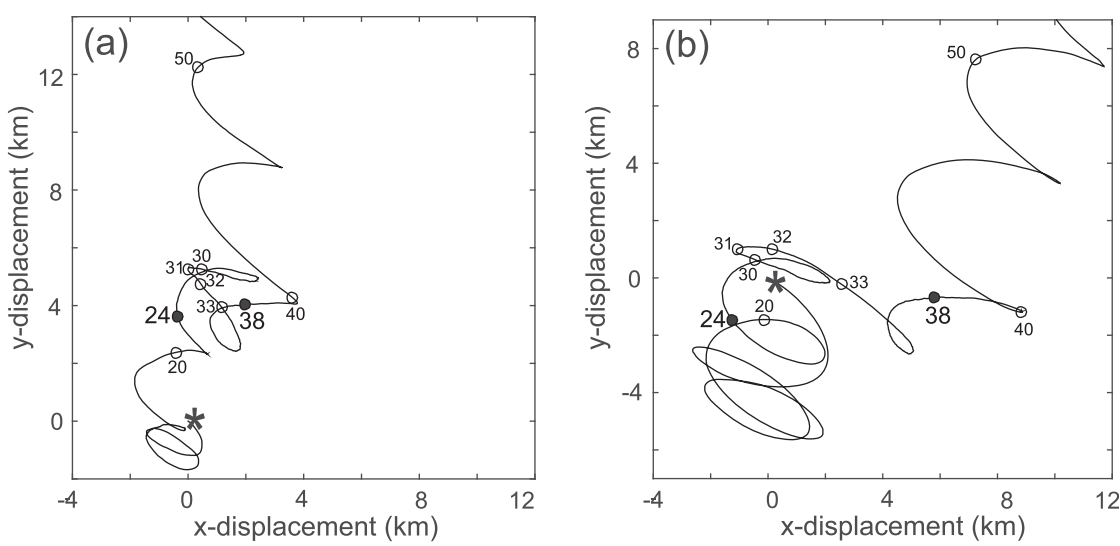

FIG. 16. Progressive vector diagrams from VACP currents measured at bin mean heights above bottom of (a) 2.66 and (b) $14.1 \mathrm{~m}$ (approximately middepth): all horizontal axes are $16 \mathrm{~km}$ in length. A star denotes the start of the first record in session 001; circles mark the start of the numbered records. Tropical Storm Barry occupied records 20-40, with full-depth backscatter present only in records 24-29 and 33-38, when net horizontal transport distances are small. Mean flows following storm passage (after record 40) are larger and roughly toward the north near bottom and toward the northeast at middepth.

energy through the drag of a solid boundary is greater than that induced at the deep ocean mixed layer base by intermittent mixed layer deepening and internal wave radiation into the interior of the deep sea.

\section{Sediment resuspension and transport during TS Barry}

Langmuir supercells were originally given the name because of the importance, perhaps dominance, of supercell events in transporting sediment long distances on shallow shelves. During extended storms on shelves with coastlines to the left of the vector wind direction, strong geostrophically balanced downwind flows (Grosch and Gargett 2016) carry resuspended sediment, some part of which has been moved out of the bottom boundary layer and throughout the water column by LS, large distances down the coast. A progressive vector diagram (PVD) from LEO (Gargett et al. 2014) documents downwind/downcoast movement by $\sim 40 \mathrm{~km}$ at middepth, and the PVD from a similar Tower R2 event with directionally constant winds (not shown) indicates even larger transport distances, nearly $60 \mathrm{~km}$ at middepth.

As seen in Fig. 17, TS currents and waves are sufficiently large that total bottom stress exceeds that required to resuspend a full size range (silt to sand) of bottom sediments throughout the period of records 24-38. The component of stress due to surface waves rises rapidly in record 24 , peaks during the first wind maximum, and then declines slowly. Although total stress also declines somewhat after the first wind peak, it remains sufficiently elevated through at least record 38 that lack of resuspended sediment cannot explain the "hole" in backscatter during records 30-31: this must instead be attributed to the absence of full-depth structures at this time, as documented above.

The latter half of the storm is observed at Tower R2 during 3 June (yearday 153), a time when a map of the SeaWiFS particulate product (Savidge et al. 2008, their Fig. 2b) shows the entire Georgia shelf "lit up" by sediment backscatter, demonstrating much wider spatial significance of the Tower R2 time series. However, the PVDs of Fig. 16 show that although resuspension may be high during the TS, there is very little mean current during times when high backscatter is present in the water column, suggesting limited net sediment transport. Despite absence of large net transport, tropical storms may affect shelf sediment transport by resorting surficial sediments, as heavier components fall out faster after storm passage, leaving the lightest fraction on the surface. As seen in Fig. 17, wave stress declines to prestorm levels by record 40 . However as a result of large stormgenerated mean flows (Fig. 16b), total stress remains above the level sufficient to resuspend silt, even periodically above that necessary to resuspend sand, for a substantial period of time after storm passage. Poststorm peaks in bottom stress, clearly associated with the semidiurnal tide, are correlated with backscatter plumes of bottom origin, plumes that diminish and eventually disappear as total stress returns to prestorm levels by session end. The poststorm period would arguably be associated with preferential net transport of the lighter sediment fractions that are available on the sediment surface due to storm resorting, as well as 

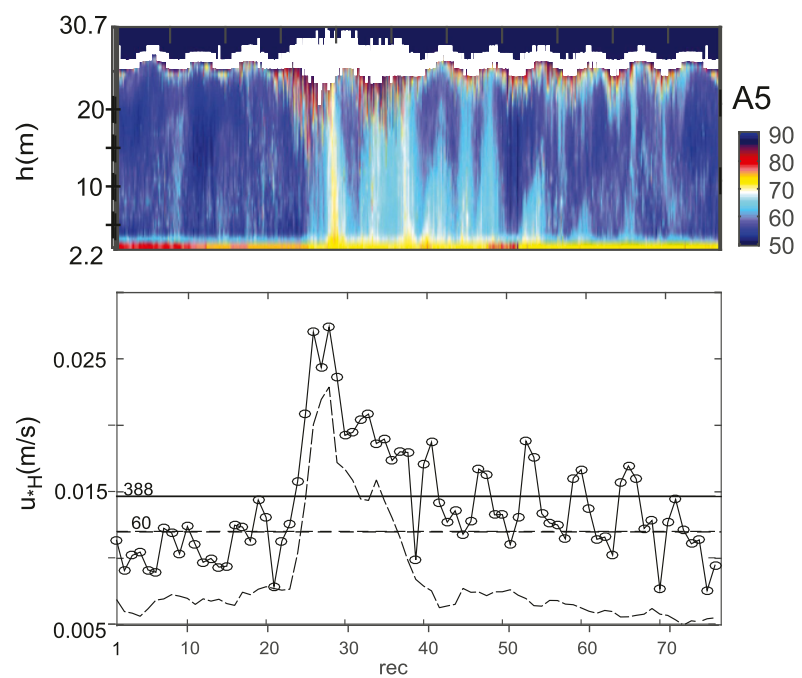

FIG. 17. (top) The backscatter amplitude over the full water column during session 001 as a function of height above bottom $h$. (bottom) Record-averaged values of bottom stress associated with the surface wave field (dashed line) and total stress (wave stress plus quadratic stress due to mean and tidal currents; solid line), with symbols plotted at the beginning of a record. Horizontal lines mark resuspension thresholds for size categories corresponding roughly to silt ( $60 \mu \mathrm{m}$; dashed) and sand (388 $\mu \mathrm{m}$; solid).

present in the water column a larger portion of the time than heavier fractions.

\section{Summary and conclusions}

In summary, we have presented the most complete observations yet available of surface waves and turbulence associated with passage of a tropical storm across a continental shelf. The description includes collocated measurements of forcing fields, both complete meteorological fields from a tower-mounted mast and directional surface wave spectra from a VADCP, the latter enabling computation of the directional Stokes function as it varies with depth and time. The VADCP also provided mean (including tidal) flows and turbulent structures in the water column. Some major databased conclusions are the following.

1) Given surface forcing magnitudes as described by the $\{\mathrm{La}, \mathrm{Ra}\}$ parameter space, full-depth velocity and backscatter structures observed in the water column during TS Barry are full-depth Langmuir cells, although

2) as documented by SG17 for directionally steady winds, LS structures observed at Tower R2 during TS passage are less organized than the archetypical Langmuir supercell structures defined by Gargett et al. (2004) based on observations at LEO.

3) Location within the LS region of the $\{\mathrm{La}, \mathrm{Ra}\}$ parameter space is a necessary but not sufficient condition for existence of LS, as evidenced by disappearance of full-depth velocity and backscatter structures for a time (encompassing records 30 and 31) between TS wind speed maxima, despite $\{\mathrm{La}, \mathrm{Ra}\}$ values remaining within or near the LS region.

The present analysis indicates that, except for the time period referred to in point 3 above, surface forcing associated with the TS changes slowly enough that the time-dependent forcing in this case produces roughly the same results as a case in which winds are essentially steady in both magnitude and direction.

A possible explanation for why LS structures are nonetheless generally less organized at Tower R2 than at LEO emerged from an analysis that examined not only $g_{*}$ [Eq. (2)], the growth rate for Langmuir circulation based on near-surface parameters (Leibovich 1977), but also a similar near-bottom growth rate $G_{*}$ [Eq. (3), adapted from that introduced by Gargett et al. (2014)] based on the idea that Langmuir forcing occurs not just at the surface but wherever there is both a source of vertical vorticity and a nonzero, vertically sheared Stokes drift velocity. Since a bottom boundary layer can provide the former and, in a shallow enough water column, surface waves of intermediate type can provide the latter, it was supposed that there would emerge a threshold value of $G_{*}$ below which near-bottom forcing would not be large enough to generate full-depth structures. What emerged instead is a distinct threshold in the surface growth rate: every LS event available to us from either LEO or Tower R2 deployments has full-depth velocity and backscatter structures in an unstratified water column if $g_{*}>g_{*}^{\text {crit }} \simeq 2 \times 10^{-3} \mathrm{~s}^{-1}$, irrespective of very different values of $G_{*}$ at the two locations. The existence of this critical value of $g_{*}$ allows estimation of how deep an unstratified water column must be before LS will not be expected: specifically, it must be deeper than $H^{\text {crit }}$ as given by Eq. (4). Although this result has been derived from coastal data, it could potentially be used to predict the maximum penetration depth of quasi-organized large eddies associated with Langmuir forcing in an open ocean surface mixed layer that is deeper than $H^{\text {crit }}$.

While Eq. (4) provides a useful predictive result, it leaves unanswered the question of why Tower R2 LS structures are less organized than those at LEO. Since $G_{*}$ does not determine existence of LS, we hypothesize that instead it determines their degree of organization. If the near-bottom growth time scale $T_{*}=G_{*}^{-1}$ is short relative to time scale(s) over which the near-bottom mean plus tidal current changes, there is time for organized near-bottom structure to emerge. The hypothesis 
that it is the time rate of change of near-bottom current, rather than its magnitude and/or direction, that determines the degree of organization of LS structures is an important subject for further investigation. We note that Sullivan et al. (2007) suggest an alternate hypothesis, reporting that an increased level of intermittent breaker forcing of their surface layer LES caused reduced spatial organization of LC. However they did not provide a quantitative measure of "organization": as well we have no direct information on breaking at either LEO or Tower R2, so it is not possible to investigate how much observed differences in organization of LS between our two coastal sites might be expected due to differences in breaking states.

The third point above is a major departure from the steady wind case. The quiescent records lie within or near the region of the $\{\mathrm{La}, \mathrm{Ra}\}$ regime diagram associated with LS. Why then are LS, nor indeed LC of any scale, not observed in these records?

Various potential explanations were dismissed by counterexample, that is, by finding a record with LS and the same forcing and/or diagnostic features of the quiescent midstorm records that lack LS. A significant result from this exploration is that, given magnitude of surface wind/wave forcing sufficient to produce $g_{*}>g_{*}^{\text {crit }}$, observed features of LS such as existence, organization, magnitude of $\overline{\left\langle w^{\prime 2}\right\rangle}$, and so on are essentially the same whether the angle between the surface Stokes drift and the wind is zero, as it is during the first half of the storm, or relatively large, $\sim 40^{\circ}-50^{\circ}$ in the second half of the storm.

We are left with two possible explanations for point 3 . First, values of $g_{*}$ for records 30 and 31 are minimal for those during the storm period: using an ad hoc estimate of the effect of observed directional difference between the required Stokes shear and the wind-driven shear, "wind-aligned" values lie nearer the critical value discussed above. Thus one explanation is that decreases in $u_{*}$ and $u_{S 0}$ combine with misalignment between Stokes shear and (wind generated) mean shear to drive a windaligned surface growth rate $g_{*}^{a}=g_{*}(\cos \theta)^{1 / 2}$ below the critical value. We have considered this explanation unlikely, as it would imply very sharp criticality in $g_{*}$, one separating a state with full-depth LC from a state with no LC, rather than a state with $\mathrm{LC}$ over a depth range less than the full depth. However the nature of this boundary should be the subject of further research.

An alternate explanation for point 3 consistent with the observations is decrease in wave breaking during the period of wind veering, resulting in decreased supply of "effective" near-surface vertical vorticity for LC generation. We are unable to explore this explanation further without necessary direct observations of sea state and urge that such observations be part of subsequent similar measurements, since the consequences are significant. However if vertical vorticity associated with wave breaking is indeed more effective for generation of LC than that associated with a stress-driven boundary layer, then the relevant input of vorticity is always at least roughly in the direction of the dominant waves, hence that of the surface Stokes drift velocity. This would imply that, provided magnitudes of surface wind/wave forcing are equivalent, wind direction may differ substantially from that of the surface Stokes drift without significantly affecting the generation/characteristics of LC, as indeed documented by our observations at the second wind maximum. This conclusion itself then implies that LES carried out with standard surface stress forcing in the wind direction (Van Roeckel et al. 2012) to investigate the effect of different angles between surface Stokes velocity and the wind should be repeated with a stochastic breaker model (e.g., Sullivan et al. 2007) that models the more effective surface input of vertical vorticity from breaking waves. In addition, the suggested importance of vertical vorticity supplied by wave breaking implies that effects of wave breaking cannot be modeled in LES solely by enhancing surface TKE flux (Kukulka and Veron 2019) but must also involve provision of effective vorticity.

Last, it was demonstrated that although LS move resuspended sediment throughout the water column during the two periods of wind maxima involved in passage of TS Barry, there is little net transport of sediment by this storm, unlike large net transports during supercell events associated with storm winds from a relatively constant direction. Tropical storms may nonetheless influence shelf sediment transport by resorting surficial sediments.

Acknowledgments. We greatly appreciate the support that Greg Dusek and Harvey Seim so graciously gave us in adapting surface wave analysis software to the VADCP data, as well as useful comments from two reviewers. We thank the National Science Foundation (author Savidge: OCE-0536326 and OCE-0926852; author Gargett: OCE-0136403 and OCE-0927724) and the National Oceanic and Atmospheric Administration (Gargett: NA06RU0139) for supporting this research. Gargett acknowledges with gratitude the profound contributions made to studies of Langmuir circulation in shallow water by her late colleague Chester Grosch.

Data availability statement. Data used in the study are archived online as follows: Tower R2 meteorological data(as station SPAG1): https://ndbc.noaa.gov); VADCP and T-chain data: https://outlookuga-my.sharepoint.com/ :f:/g/personal/skidaway_uga_edu/Ejn5U28DNf1OgVaA9 PlnhR8BtyKhhvaAzmh7W1mrmcnvZw?e=GPDLKK. 


\section{APPENDIX A}

\section{Computation of Directional Wave Spectra from VADCP Data}

To appreciate the frequency (wavenumber) ranges in which various measured fields are most useful in sampling surface wave height, consider the response functions of Fig. A1, providing (time) mean square wave heights from mean square values of fluctuation pressure $\left\langle p^{2}\right\rangle$, horizontal velocity $\left\langle u^{2}\right\rangle$, and vertical velocity $\left\langle w^{2}\right\rangle$ (Phillips 1977) for a single wave of wavenumber $k$, related to frequency $\omega$ by the dispersion relation $\omega^{2}=$ $g k \tanh k H$, where $H$ is water column depth. An ideal combination of sensors would be pressure for low frequencies and vertical velocity for extension to higher frequencies. However, because the VADCP was not collocated with the pressure sensor (and the slant beams of the VADCP are necessary for directional computations), our calculations of directional spectra use only velocity data.

Because velocities associated with surface waves decrease with distance of the measurement from the surface, we use the shallowest depths for which each type of velocity data is available. For an individual 2-h record, the surface bin SB at each acoustic ping is identified in vertical-beam backscatter. Data for $w$ are taken from wbin $=\min (\mathrm{SB})-1$, that is, one bin below the minimum of SB over the record. Data for slant-beam velocities are taken from sbin and sbin -1 , where sbin is the shallowest bin that reliably avoids sidelobe contamination from the instantaneous surface. For record 27, these values are (wbin, sbin, sbin -1$)=(19,14,13)$, corresponding to bin mean heights of $(19.8,15.0,14.1) \mathrm{m}$ above bottom, hence depths of $(5.8,10.6,11.5) \mathrm{m}$ in a 25.6 -m-deep water column. Once data have been selected, bad values are replaced using vertical interpolation and a linear least squares fit is removed from all fields.

Johnson (2002) uses the time series of a first input data type to generate a frequency spectrum, then cross spectra of all data inputs to generate directional spreading. However because radial velocity data from an ADCP is noisy compared with data from, say, a pressure sensor, use of averages generally produces a more accurate initial spectrum. Fig. A2 displays frequency spectra calculated with three choices of data fields: all (eight) selected slant-beam bins (Fig. A2a), only the vertical-beam bin (Fig. A2b), and the average of all selected slant-beam bins plus the vertical-beam bin (Fig. A2c). All spectra and cross spectra are estimated using a direct Fourier transform method, as exploration showed that other popular methods produce larger noise at higher frequencies associated

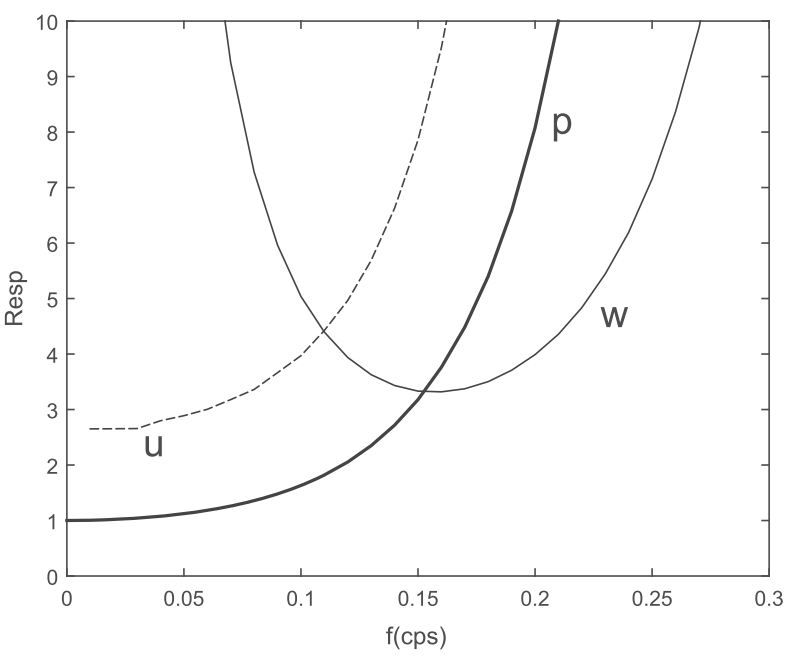

FIG. A1. Response functions (resp) calculated for various input fields: $p$ (thick solid), $u$ (dashed), and $w$ (thin solid). For session 001, record 27, height above bottom of mean surface $H=25.6 \mathrm{~m}$, the height of the pressure sensor $=19.1 \mathrm{~m}$, the height of the verticalbeam bin $=19.8 \mathrm{~m}$, and the height of the shallowest slant-beam bin $=15.0 \mathrm{~m}$. For the record-average depth $H$, sensor depths are $6.5 \mathrm{~m}$ for $p, 5.8 \mathrm{~m}$ for $w$, and $10.6 \mathrm{~m}$ for $u$.

with significant contributions to the Stokes velocity integral. Final results are smoothed by a three-point Hanning filter in both frequency and direction.

Figure A2b shows that use of only the vertical beam results in a frequency spectrum with a large low-frequency peak, due to the extreme response correction for $w$ as $f \rightarrow 0$, plus overall higher levels, resulting partly from improved sensitivity to higher-frequency waves because this "sensor" is nearest the surface and partly due to larger noise in the single time series. In contrast, Figs. A2a and A2c are nearly identical (Fig. A2c has a small residual peak near zero frequency and very slightly larger SWH, associated with use of the vertical-beam data as one of nine independent estimates): both are essentially identical to the frequency spectrum (not shown) from the tower-mounted pressure sensor.

Use of vertical-beam data is desirable not only because it provides data nearer the surface than slant beams, but also because it doubles the horizontal resolution of the Doppler "array." Figure A3 presents directional spectra for the three cases of Fig. A2 as logarithmic (base 10) displays. Using only slant beams (Fig. A3a) results in a " $180^{\circ}$ ghost" at frequencies around $f_{g} \sim 0.2 \mathrm{~Hz}$. Inability of slant-beam data to distinguish between $\pm 180^{\circ}$ near $f_{g}$ can be understood in terms of the horizontal aperture associated with the slant beams. If $L_{13}$ is the distance between slant beams 1 and 3 at the height above bottom $h_{13}=14.1 \mathrm{~m}$ of bin 13 (the deepest bin used for slant beams and hence the smallest aperture), then $L_{13}=2 h_{13} \tan 30^{\circ}=16.3 \mathrm{~m}$. 

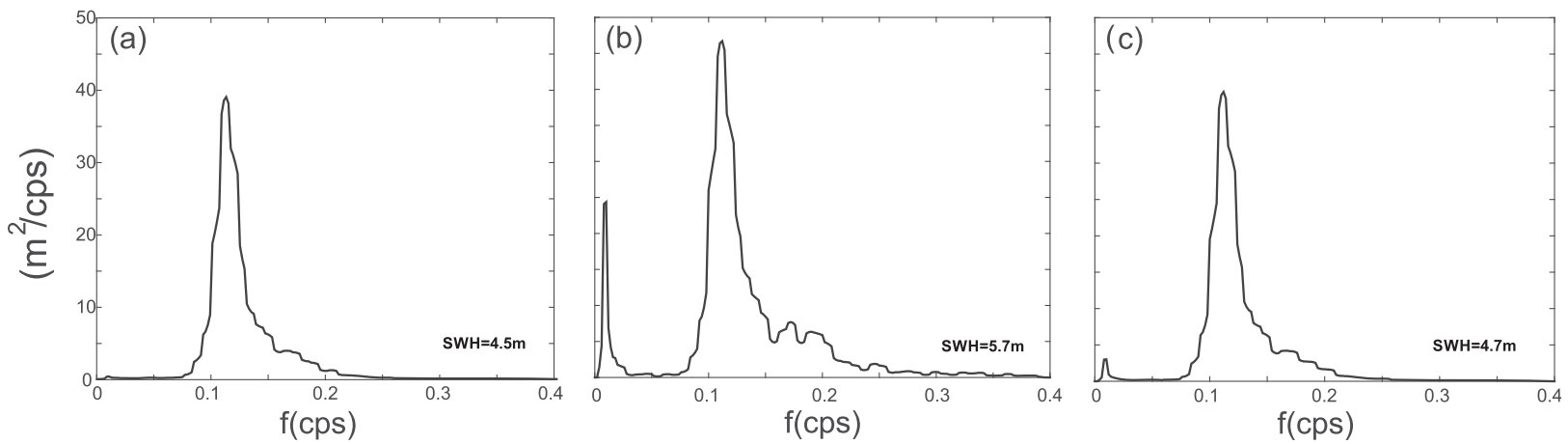

FIG. A2. Frequency spectra of surface displacement calculated using data from (a) eight slant-beam bins only and the same slant-beam data plus

(b) one bin of vertical velocity data as the first field in the data list and (c) the same bin of vertical velocity as the last field in the data list.

A wave with a length of 2 times this value has an associated wavenumber of $k=2 \pi /\left(2 L_{13}\right)=0.19 \mathrm{rad} \mathrm{m}^{-1}$ and associated frequency of $1.36 \mathrm{rad} \mathrm{s}^{-1}$, or $0.21 \mathrm{cps}$. When such surface waves propagate normal to the plane containing beams 1 and 3, the slant-beam sensors will return a fixed phase of $180^{\circ}$ and hence be unable to reliably determine the actual propagation direction. Addition of one bin of data from the vertical beam provides cross correlations at half the slant-beam aperture, serving to identify the actual direction more accurately and effectively eliminating the $180^{\circ}$ ghost at $f_{g}$ (Fig. A3c).

\section{APPENDIX B}

\section{Scores for Structural Organization of LS}

It is useful to quantify the concept of structural organization of LS structures, that is, the degree to which full-depth turbulent velocity fields exhibit organized characteristics of LS as cartooned in Fig. 3 of Gargett et al. (2004). Major features are 1) coincidence of regions of downward/upward vertical velocity with those of enhanced near-bottom downwind/upwind velocity, and 2) change in sign of near-bottom crosswind velocity at the center of the downwelling/upwelling.

Organization will be quantified by "scores" that attempt to estimate how well observations fulfill these two requirements. Fluctuating velocity fields from a single record are first heavily smoothed by Hanning filters in both time ( $6 \mathrm{~min}$ ) and the vertical (three bins) dimension, and horizontal velocities are rotated to wind coordinates, producing fields such as those depicted in Fig. B1: $u$ is alongwind velocity and $v$ is crosswind velocity. The vertical velocity field is then averaged over depth using the maximum vertical extent available over the time extent of a record: this time series $(\bar{w})$ is overplotted on the field of $w$ in Fig. B1b. In the marked (a)

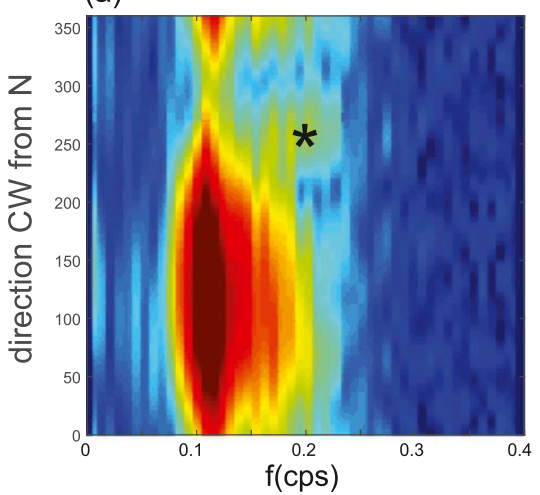

(b)

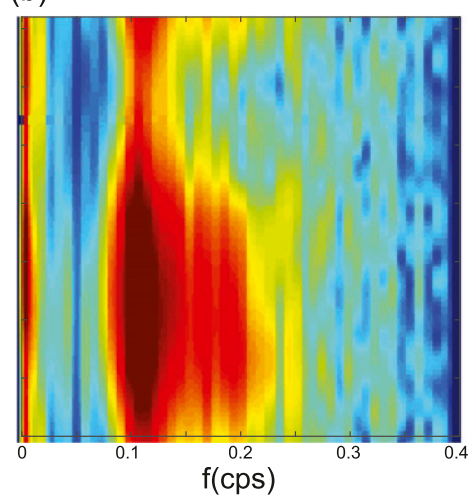

(c)

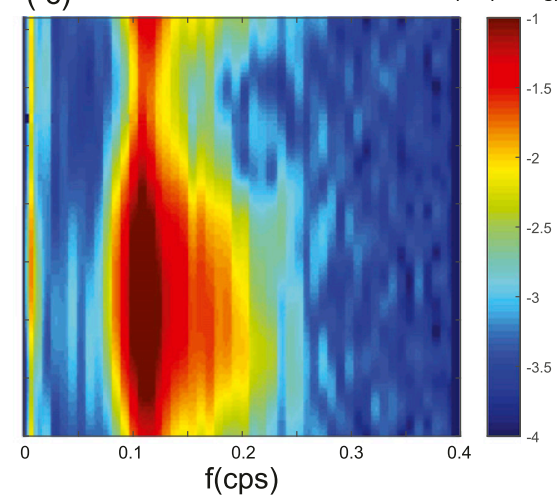

FIG. A3. Directional wave spectra (note the logarithmic scale) computed for the three cases of Fig. A2: directions are from which the waves are propagating. Note the $180^{\circ}$ ghost (marked by the star) in (a) and the increased noise over all frequencies in (b). Use of all slantbeam data to produce the initial frequency spectrum substantially decreases this noise, whereas addition of the vertical-beam data extends the measurements to somewhat higher wavenumbers and, by doubling the spatial resolution of the array, effectively eliminates the $180^{\circ}$ ghost. 


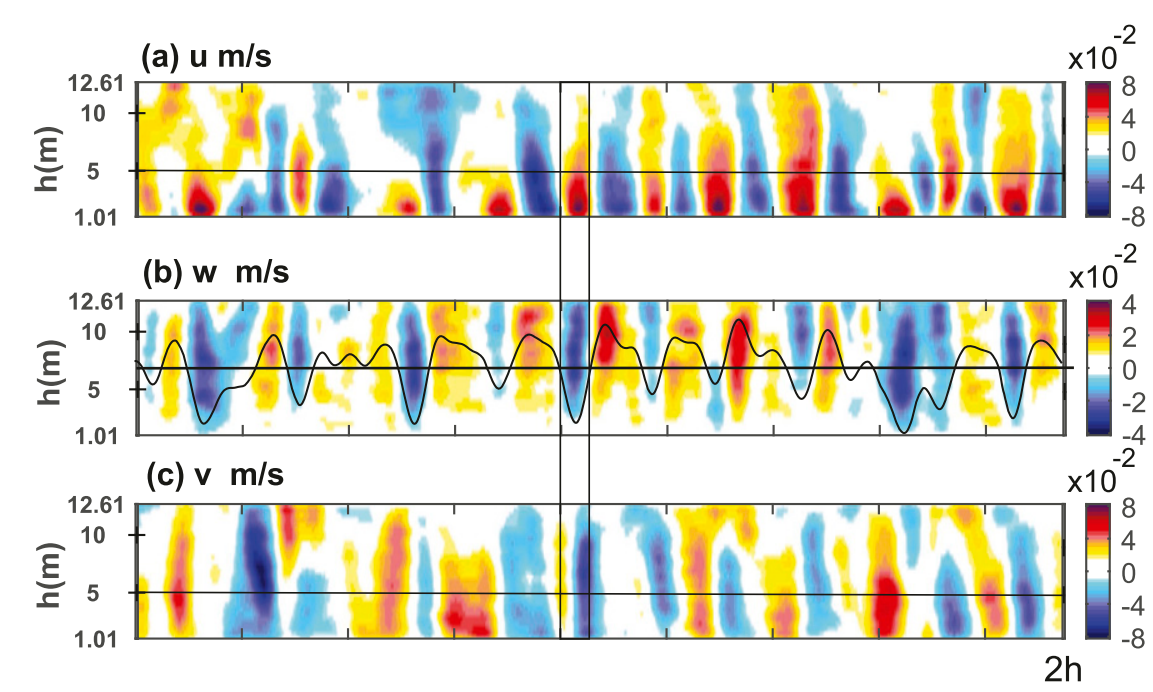

FIG. B1. Fields of smoothed fluctuating velocities used in calculating the degree to which observed structures possess the characteristics of prototypical LS at LEO, shown here. The time series of vertically averaged fluctuation vertical velocity $\bar{w}$, superimposed on the $w$ field of (b), is used to define periods of downwelling and upwelling, within which scores for the behavior of the $u$ and $v$ fields, respectively in the wind and crosswind directions, are defined using data within $5 \mathrm{~m}$ of the bottom [horizontal lines in (a) and (c)].

downwelling, note the two features mentioned: $u$, horizontal velocity parallel to the wind, is positive (downwind), whereas crosswind velocity $v$ changes sign in the middle of the downwelling (the direction of the sign change is determined by the sign of crosswind mean velocity at the time but is unimportant to the metric that will be defined for this field). Zero-crossings of $\bar{w}$ are used to identify the time extents of individual downwellings and upwellings, and two metrics are computed for each. Since the two characteristics described above are most pronounced near the bottom, the metrics use values of $u$ and $v$ from the bottom $5 \mathrm{~m}$ of the water column, that is, below the horizontal lines in Figs. B1a and B1c.

First consider the simplest case of the wind-parallel velocity. A time interval corresponding to a single downwelling/upwelling feature is awarded uscore $=1$ if $\langle u\rangle$, the time mean of $u$ over the interval, has the correct sign, for example, if within a downwelling, $\langle u\rangle$ is positive (downwind). The final Uscore is the average of the uscore values over all features within the record.
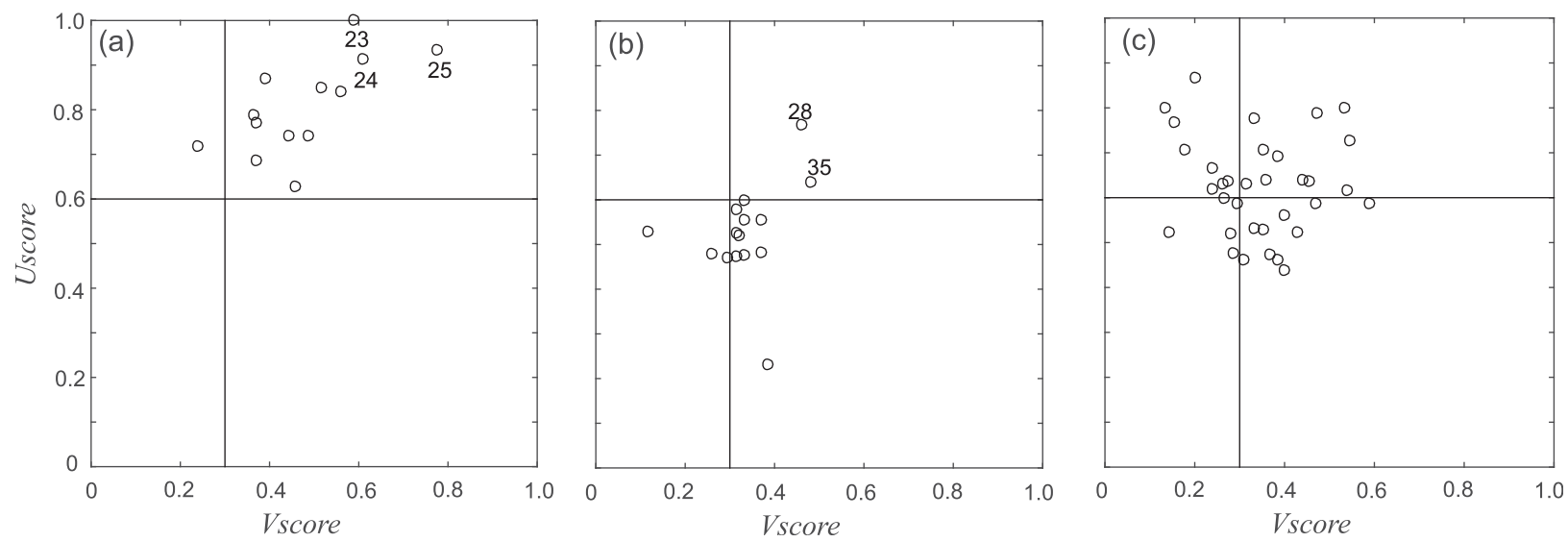

FIG. B2. The Uscore plotted against Vscore for (a) the prototype LS event at LEO (record 24, shown in Fig. B1) and records with fulldepth velocity and backscatter signals from Tower R2 during (b) the TS (session 001) with varying wind direction and (c) an LS event (session 017) with stable wind direction. During the TS, the only scores comparable to those characteristic of LEO are from records 28 and 35 , near the respective peaks of wind forcing. 
The change of sign in crosswind velocity is harder to quantify. A change of sign perfectly centered within a feature would have Vneg/Vpos $=0.5 / 0.5=1$, where Vneg and Vpos are respectively the number of measurements within the feature with $v \leq 0$ and $v>0$. However, even well-organized features may, for example, have a slight tilt with depth, resulting in unequal numbers of positive and negative values of $v$. The metric we have devised requires there to be "significant" numbers of both positive and negative values of $v$ within the feature, awarding vscore $=1$ if neither Vneg nor Vpos fall below 0.25 of the length of the feature. Again, the final Vscore is the average of the vscore values over all the features within the record.

Figure B2a shows that archetypical LS records have Uscore $>0.6$ and Vscore $>0.3$ : other LS records from LEO, not shown, are similar. Of the 15 TS records in Fig. B2b, only two, 28 and 35, have similarly high values of both scores, suggesting structures that are generally less organized than those of archetypical LS. The same conclusion holds for records from Tower R2 session 017, during which wind direction was stable; here only onethird have organized scores (Fig. B2c).

\section{REFERENCES}

Banner, M. L., A. V. Babanin, and I. R. Young, 2000: Breaking probability for dominant waves on the sea surface. J. Phys. Oceanogr., 30, 3145-3160, https://doi.org/10.1175/15200485(2000)030<3145:BPFDWO > 2.0.CO;2.

Chen, S. S., J. F. Price, W. Zhao, M. A. Donelan, and E. J. Walsh, 2007: The CBLAST-hurricane program and the next-generation fully coupled atmosphere-wave-ocean models for hurricane research and prediction. Bull. Amer. Meteor. Soc., 88, 311-318, https://doi.org/10.1175/BAMS-88-3-311.

Craik, A. D. D., and S. Leibovich, 1976: A rational model for Langmuir circulations. J. Fluid Mech., 73, 401-426, https:// doi.org/10.1017/S0022112076001420.

D'Asaro, E. A., 2003: The ocean boundary layer below Hurricane Dennis. J. Phys. Oceanogr., 33, 561-579, https://doi.org/ 10.1175/1520-0485(2003)033<0561:TOBLBH >2.0.CO;2.

Gargett, A. E., and J. R. Wells, 2007: Langmuir turbulence in shallow water: Part I. Observations. J. Fluid Mech., 576, 27-61, https://doi.org/10.1017/S0022112006004575.

_- and C. E. Grosch, 2014: Turbulence process domination under the combined forcings of wind stress, the Langmuir vortex force, and surface cooling. J. Phys. Oceanogr., 44, 4467, https://doi.org/10.1175/JPO-D-13-021.1.

_ - J. R. Wells, A. E. Tejada-Martínez, and C. E. Grosch, 2004: Langmuir supercells: A mechanism for sediment resuspension and transport in shallow seas. Science, 306, 1925-1928, https:// doi.org/10.1126/science.1100849.

— A. E. Tejada-Martínez, and C. E. Grosch, 2008: Measuring turbulent large-eddy structures with an ADCP. 1. Vertical velocity variance. J. Mar. Res., 66, 157-189, https://doi.org/ 10.1357/002224008785837149.

,-- , and -2009 : Measuring turbulent large-eddy structures with an ADCP. 2. Horizontal velocity variance. J. Mar. Res., 67, 569-595, https://doi.org/10.1357/002224009791218823.
_ D. K. Savidge, and J. R. Wells, 2014: Anatomy of a Langmuir supercell event. J. Mar. Res., 72, 127-163, https://doi.org/ 10.1357/002224014814901976.

Grosch, C. E., and A. E. Gargett, 2016: Why do LES of Langmuir supercells not include rotation? J. Phys. Oceanogr., 46, 35953597, https://doi.org/10.1175/JPO-D-16-0092.1.

Holtslag, A. A. M., and F. T. M. Nieuwstadt, 1986: Scaling the atmospheric boundary layer. Bound.-Layer Meteor., 36, 201209, https://doi.org/10.1007/BF00117468.

Hwang, P. A., and E. J. Walsh, 2018: Propagation directions of ocean surface waves inside tropical cyclones. J. Phys. Oceanogr., 48, 1495-1511, https://doi.org/10.1175/JPO-D18-0015.1.

Johnson, D., 2002: DIWASP, a directional wave spectra toolbox for MATLAB: User manual. Centre for Water Research, University of Western Australia Research Rep. WP-1601-DJ (V1.1), $18 \mathrm{pp}$.

Kenyon, K. E., 1969: Stokes drift for random gravity waves. J. Geophys. Res., 74, 6991-6994, https://doi.org/10.1029/ JC074i028p06991.

Kukulka, T., and F. Veron, 2019: Lagrangian investigation of wave-driven turbulence in the ocean surface boundary layer. J. Phys. Oceanogr., 49, 409-429, https://doi.org/10.1175/JPOD-18-0081.1.

, A. J. Plueddemann, J. H. Trowbridge, and P. P. Sullivan, 2010: Rapid mixed layer deepening by the combination of Langmuir and shear instabilities: A case study. J. Phys. Oceanogr., 40, 2381-2400, https://doi.org/10.1175/2010JPO4403.1.

Leibovich, S., 1977: Convective instability of stably stratified water in the ocean. J. Fluid Mech., 82, 561-581, https://doi.org/ 10.1017/S0022112077000846.

, 1983: The form and dynamics of Langmuir circulations. Annu. Rev. Fluid Mech., 15, 391-427, https://doi.org/10.1146/ annurev.fl.15.010183.002135.

Li, M., and C. Garrett, 1997: Mixed layer deepening due to Langmuir circulation. J. Phys. Oceanogr., 27, 121-132, https://doi.org/ 10.1175/1520-0485(1997)027<0121:MLDDTL > 2.0.CO;2.

Liu, J., J.-H. Liang, J. C. McWilliams, P. P. Sullivan, Y. Fan, and Q. Chen, 2018: Effect of planetary rotation on oceanic surface boundary layer turbulence. J. Phys. Oceanogr., 48, 2057-2080, https://doi.org/10.1175/JPO-D-17-0150.1.

MacKinnon, J. A., and M. C. Gregg, 2005: Near-inertial waves on the New England shelf: The role of evolving stratification, turbulent dissipation, and bottom drag. J. Phys. Oceanogr., 35, 2408-2424, https://doi.org/10.1175/JPO2822.1.

Phillips, O. M., 1977: The Dynamics of the Upper Ocean. 2nd ed. Cambridge University Press, $336 \mathrm{pp}$.

Rabe, T. J., T. Kukulka, I. Ginis, T. Hara, B. G. Reichl, E. A. D'Asaro, R. R. Harcourt, and P. P. Sullivan, 2015: Langmuir turbulence under Hurricane Gustav (2008). J. Phys. Oceanogr., 45, 657-677, https://doi.org/10.1175/JPO-D-14-0030.1.

Sanford, T. B., J. F. Price, and J. B. Girton, 2011: Upper-ocean response to Hurricane Frances (2004) observed by profiling EM-APEX floats. J. Phys. Oceanogr., 41, 1041-1056, https:// doi.org/10.1175/2010JPO4313.1.

Savidge, D. K., and A. E. Gargett, 2017: Langmuir supercells on the middle shelf of the South Atlantic Bight: 1. Cell structure. J. Mar. Res., 75, 49-79, https://doi.org/10.1357/002224017821352641.

Savidge, W. B., A. Gargett, R. A. Jahnke, J. R. Nelson, D. K. Savidge, R. T. Short, and G. Voulgaris, 2008: Forcing and dynamics of seafloor-water column exchange on a broad continental shelf. Oceanography, 21, 179-184, https://doi.org/ 10.5670/oceanog.2008.16. 
Seim, H. E., 2000: Implementation of the South Atlantic Bight synoptic offshore observational network. Oceanography, 13, 18-23, https://doi.org/10.5670/oceanog.2000.29.

Sherwood, C. R., J. R. Lacey, and G. Voulgaris, 2006: Shear velocity estimates on the inner shelf off Grays Harbor, Washington, USA. Cont. Shelf Res., 26, 1995-2018, https://doi.org/10.1016/ j.csr.2006.07.025.

Sternberg, R. W., 1968: Friction factors in tidal channels with differing bed roughness. Mar. Geol., 6, 243-260, https://doi.org/ 10.1016/0025-3227(68)90033-9.

Sullivan, P. P., J. C. McWilliams, and W. K. Melville, 2004: The oceanic boundary layer driven by wave breaking with stochastic variability. Part I. Direct numerical simulations. J. Fluid Mech., 507, 143-174, https://doi.org/10.1017/S0022112004008882.

, — , and - 2007: Surface gravity wave effects in the oceanic boundary layer: Large-eddy simulation with vortex force and stochastic breakers. J. Fluid Mech., 593, 405-452, https://doi.org/10.1017/S002211200700897X.

, L. Romero, J. C. McWilliams, and W. K. Melville, 2012: Transient evolution of Langmuir turbulence in ocean boundary layers driven by hurricane winds and waves. J. Phys. Oceanogr., 42, 1959-1980, https://doi.org/10.1175/ JPO-D-12-025.1.

Van Roeckel, L. P., B. Fox-Kemper, P. P. Sullivan, P. E. Hamlington, and R. S. Haney, 2012: The form and orientation of Langmuir cells for misaligned winds and waves. J. Geophys. Res., 117, C05001, https://doi.org/10.1029/2011JC007516.

Wang, D. T., T. Kukulka, B. G. Reichl, T. Hara, I. Ginis, and P. P. Sullivan, 2018: Interaction of Langmuir turbulence and inertial currents in the ocean surface boundary layer under tropical cyclones. J. Phys. Oceanogr., 48, 1921-1940, https:// doi.org/10.1175/JPO-D-17-0258.1. 